\title{
First-principles scattering matrices for spin transport
}

\author{
K. Xia,* M. Zwierzycki, ${ }^{\dagger}$ M. Talanana, and P. J. Kelly \\ Faculty of Science and Technology and MESA ${ }^{+}$Institute for Nanotechnology, University of Twente, \\ P.O. Box 217, 7500 AE Enschede, The Netherlands \\ G. E. W. Bauer \\ Kavli Institute of NanoScience, Delft University of Technology, Lorentzweg 1, 2628 CJ Delft, The Netherlands
}

(Received 15 August 2005; published 17 February 2006)

\begin{abstract}
Details are presented of an efficient formalism for calculating transmission and reflection matrices from first principles in layered materials. Within the framework of spin density functional theory and using tight-binding muffin-tin orbitals, scattering matrices are determined by matching the wave functions at the boundaries between leads which support well-defined scattering states, and the scattering region. The calculation scales linearly with the number of principal layers $N$ in the scattering region and as the cube of the number of atoms $H$ in the lateral supercell. For metallic systems for which the required Brillouin zone sampling decreases as $H$ increases, the final scaling goes as $H^{2} N$. In practice, the efficient basis set allows scattering regions for which $H^{2} N \sim 10^{6}$ to be handled. The method is illustrated for $\mathrm{Co} / \mathrm{Cu}$ multilayers and single interfaces using large lateral supercells (up to $20 \times 20$ ) to model interface disorder. Because the scattering states are explicitly found, "channel decomposition" of the interface scattering for clean and disordered interfaces can be performed.
\end{abstract}

DOI: 10.1103/PhysRevB.73.064420

PACS number(s): 75.47.De, 72.10.Bg, 72.25.Ba

\section{INTRODUCTION}

One of the most important driving forces in condensed matter physics in the last 30 years has been the controlled growth of layered structures so thin that interface effects dominate bulk properties and quantum size effects can be observed. In doped semiconductors, the large Fermi wavelength of mobile charge carriers made it possible to observe finite size effects for layer thicknesses on a micron scale. Much thinner layers must be used in order to make such observations in metals because Fermi wavelengths are typically of the order of an interatomic spacing. Nevertheless, following rapidly on the heels of a number of important discoveries in semiconductor heterostructures, interfacedominated effects such as interface magnetic anisotropy, oscillatory exchange coupling, and giant magnetoresistance (GMR) were found in artificially layered transition metal materials. Reflecting the shorter Fermi wavelength, the characteristic length scale is of order of nanometers.

Our main purpose in this paper is to give details of a scheme we have developed which is suitable for studying mesoscopic transport in inhomogeneous, mainly layered, transition metal magnetic materials. In the context of a large number of schemes designed to study transport either from first principles ${ }^{1-18}$ or based upon electronic structures calculated from first principles ${ }^{19-24}$ we will require our computational scheme to be (i) physically transparent, (ii) first principles, requiring no free parameters, (iii) capable of handling complex electronic structures characteristic of transition metal elements, and (iv) very efficient in order to be able to handle lateral supercells to study layered systems with different lattice parameters and to model disorder very flexibly. A tight-binding (TB) muffin-tin-orbital (MTO) implementation of the Landauer-Büttiker formulation of transport theory within the local-spin-density approximation (LSDA) of density-functional theory (DFT) will satisfy these requirements.
Because wave transport through interfaces is naturally described in terms of transmission and reflection, the LandauerBüttiker (LB) transmission matrix formulation of electron transport gained rapid acceptance as a powerful tool in the field of mesoscopic physics, ${ }^{25,26}$ once the controversies surrounding the circumstances under which different expressions should be used had been resolved. ${ }^{25}$ The two-terminal conductance of a piece of material is measured by attaching leads on either side, passing a current through these leads, and measuring the potential drop across the scattering region. In the LB formulation of transport theory, the conductance $G$ is expressed in terms of a transmission matrix $t \equiv t\left(E_{F}\right)$

$$
G=\frac{e^{2}}{h} \operatorname{Tr}\left\{t t^{\dagger}\right\}
$$

where the element $t_{\mu \nu}$ is the probability amplitude that a state $|\nu\rangle$ in the left-hand lead incident on the scattering region from the left (see Fig. 1) is scattered into a state $|\mu\rangle$ in the right-hand lead. The trace simply sums over all incident and transmitted "channels" $\nu$ and $\mu$ and $e^{2} / h$ is the fundamental unit of conductance. In much current work on first-principles transport the conductance is calculated directly from Green's functions expressed in some convenient localized orbital representation. ${ }^{27}$ Explicit calculation of the scattering states is avoided by making use of the invariance properties of a trace. Because we want to make contact with a large body of theoretical literature ${ }^{28}$ on mesoscopic physics and address a wider range of problems in the field of spin-dependent transport, we will calculate the microscopic transmission and reflection matrices $t$ and $r$. By using a real energy, we will avoid the problems encountered in distinguishing propagating and evanescent states when a small but finite imaginary part of the energy is used. The Landauer-Büttiker formalism satisfies our first requirement of physical transparency. 


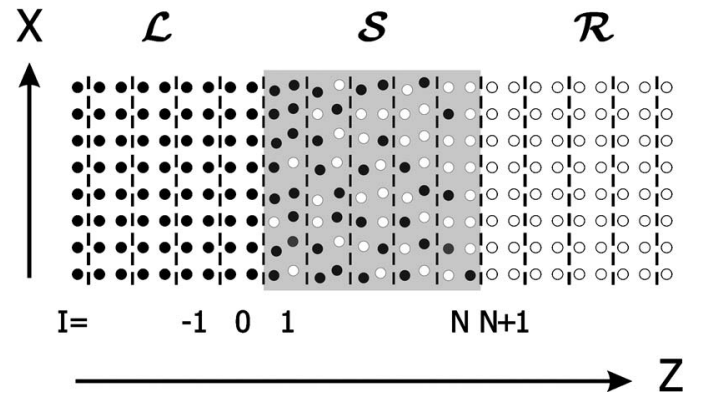

FIG. 1. Sketch of the configuration used in the LandauerBüttiker transport formulation to calculate the two terminal conductance. A (shaded) scattering region $(\mathcal{S})$ is sandwiched by left- $(\mathcal{L})$ and right-hand $(\mathcal{R})$ leads which have translational symmetry and are partitioned into principal layers perpendicular to the transport direction. The scattering region contains $N$ principal layers but the structure and chemical composition are in principle arbitrary.

In developing a scheme for studying transport in transition metal multilayers, a fundamental difference between semiconductors and transition metals must be recognized. Transition metal atoms have two types of electrons with different orbital character. The $s$ electrons are spatially quite extended and, in solids, form broad bands with low effective masses; they conduct easily. The $d$ electrons are much more localized in space, form narrow bands with large effective masses, and are responsible for the magnetism of transition metal elements. The "magnetic" electrons, however, being itinerant do contribute to electrical transport. The appropriate framework for describing metallic magnetism, even for the late $3 d$ transition metal elements, is band theory. ${ }^{29}$ An extremely successful framework exists for treating itinerant electron systems from first principles and this is the local density approximation (LDA) of density-functional theory (DFT). For band magnetism, the appropriate extension to spin-polarized systems, the local spin-density approximation (LSDA) satisfies our second requirement of requiring no free parameters. ${ }^{30}$

Oscillatory exchange coupling in layered magnetic structures was discussed by Bruno in terms of generalized reflection and transmission matrices ${ }^{31}$ which were calculated by Stiles $^{32-34}$ for realistic electronic structures using a scheme ${ }^{35,36}$ based on linearized augmented plane waves (LAPWs). At an interface between a nonmagnetic and a magnetic metal, the different electronic structures of the majority and minority spin electrons in the magnetic material give rise to strongly spin-dependent reflection. ${ }^{1,37}$ Schep used transmission and reflection matrices calculated from first-principles with an embedding surface Green's function method $^{38}$ to calculate spin-dependent interface resistances for specular $\mathrm{Co} / \mathrm{Cu}$ interfaces embedded in diffusive bulk material. ${ }^{4}$ The resulting good agreement with experiment indicated that interface disorder is less important than the spindependent reflection and transmission from a perfect interface. Calculations of domain wall resistances as a function of the domain wall thickness illustrated the usefulness of calculating the full scattering matrix. ${ }^{6,39}$ However, the LAPW basis set used by Stiles and Schep was computationally too expensive to allow repeated lateral supercells to be used to model interfaces between materials with very different, in- commensurate lattice parameters or to model disorder. This is true of all plane-wave based basis sets which typically require of order 100 plane waves per atom in order to describe transition metal atom electronic structures reasonably well.

Muffin-tin orbitals (MTO) form a flexible, minimal basis set leading to highly efficient computational schemes for solving the Kohn-Sham equations of DFT. ${ }^{40-43}$ For the close packed structures adopted by the magnetic materials $\mathrm{Fe}, \mathrm{Co}$, $\mathrm{Ni}$, and their alloys, a basis set of nine functions $(s, p$, and $d$ orbitals) per atom in combination with the atomic sphere approximation (ASA) for the potential leads to errors in describing the electronic structure which are comparable to the absolute errors incurred by using the local density approximation. This should be compared to typically 100 basis functions per atom required by the more accurate LAPW method. MTOs thus satisfy our third and fourth requirements of being able to treat complex electronic structures efficiently.

The tight-binding linearized muffin-tin-orbital (TBLMTO) surface Green's function (SGF) method has been developed to study the electronic structure of interfaces and other layered systems. When combined with the coherentpotential approximation (CPA), it allows the electronic structure, charge, and spin densities of layered materials with substitutional disorder to be calculated self-consistently very efficiently. ${ }^{44}$ In this paper we describe how we have combined a method for calculating transmission and reflection matrices based on wave-function matching (WFM), in a form given by $\mathrm{Ando}^{45}$ for an empirical tight-binding Hamiltonian, with a first-principles TB-MTO basis. ${ }^{42}$ Applications of the method to a number of problems of current interest in spin-transport have already been given in a number of short publications: to the calculation of spin-dependent interface resistances where interface disorder was modeled by means of large lateral supercells; $;{ }^{9}$ to the first principles calculation of the so-called mixing conductance parameter entering theories of current-induced magnetization reversal ${ }^{46}$ and Gilbertdamping enhancement via spin-pumping; ${ }^{47}$ to a generalized scattering formulation of the suppression of Andreev scattering at a ferromagnetic/superconducting interface; ${ }^{48}$ and to the problem of how spin-dependent interface resistances influence spin injection from a metallic ferromagnet into a III-V semiconductor. ${ }^{49-51}$ These examples amply demonstrate that the fourth requirement is well-satisfied.

In Sec. II, we give technical details of the formalism and illustrate it in Sec. III where we calculate the transmission matrices for clean and disordered $\mathrm{Co} / \mathrm{Cu}$ interfaces, document a number of convergence and accuracy issues and give a detailed "channel-decomposition" analysis of the scattering in the presence of disorder. A comparison with other methods is made in Sec. IV.

\section{THEORY}

Central to the wave-function matching method for calculating the transmission and reflection matrices is the equation of motion (EOM) for electrons with energy $\varepsilon$, relating the vectors of coefficients $\mathbf{C}_{I}$ for layers $I-1, I$, and $I+1$ : 


$$
\mathcal{H}_{I, I-1} \mathbf{C}_{I-1}+(\mathcal{H}-\varepsilon)_{I, I} \mathbf{C}_{I}+\mathcal{H}_{I, I+1} \mathbf{C}_{I+1}=0 .
$$

Here, $\mathbf{C}_{I} \equiv C_{I i}$ describes the wave-function amplitude in terms of some localized orbital basis $|i\rangle$ of dimension $M$ where $i$ labels the atomic orbital and atom site. [For the muffin-tin orbitals to be outlined in Sec. II A, $i$ will be a combined index $\mathbf{R} l m$, where $l$ and $m$ are the azimuthal and magnetic quantum numbers, respectively, of the MTO defined for an atomic-spheres-approximation (ASA) potential on the site R.] The EOM does not restrict us to only considering nearest-neighbor interactions since atoms can always be grouped into layers defined as to be so thick that the interactions between layers $I$ and $I \pm 2$ are negligible (see Fig. 1). Such layers are called principal layers. Their thickness depends on the range of the interactions which in turn partly depends on the spatial extent of the orbital basis. It will be minimized by using the highly localized tight-binding MTO representation.

Consider the situation sketched in Fig. 1 where the scattering region $\mathcal{S}$ is contacted with left $(\mathcal{L})$ and right $(\mathcal{R})$ leads which have perfect lattice periodicity and support welldefined scattering states. We assume that the ground state charge and spin densities and the corresponding Kohn-Sham independent electron potential have already been calculated self-consistently. The calculation of the scattering matrix can now be split into two distinct parts. In the first stage, to be discussed in Sec. II B, the eigenmodes of the leads $\mathbf{u}_{\mu}\left(=\mathbf{C}_{0}\right.$ for the $\mu$ th mode), of which there are $2 M$, are calculated using an EOM appropriate to MTOs and making use of the lattice periodicity. By calculating their $\mathbf{k}$ vectors (which are in general complex) and velocities $v_{\mathrm{k}}$, the eigenstates can be classified as being either left-going $\mathbf{u}_{\mu}(-)$ or right-going $\mathbf{u}_{\mu}(+)$. They form a basis in which to expand any left- and right-going waves and have the convenient property that their transformation under a lattice translation in the leads is easily calculated using Bloch's theorem (with $\mathbf{k}$ complex). We use the small Roman letters $i, j$ to label the nonorthogonal basis and the small Greek letters $\mu, \nu$ to label the lead eigenmodes.

In the second stage discussed in Sec. II C, a scattering region $\mathcal{S}$ is introduced in the layers $1 \leqslant I \leqslant N$ which mixes left- and right-going lead eigenmodes. The scattering region can be a single interface, a complex multilayer, a tunnel junction, etc., and the scattering can be introduced by disorder or simply by discontinuities in the electronic structure at interfaces. The $\nu \rightarrow \mu$ element of the reflection matrix, $r_{\mu \nu}$, is defined in terms of the ratio of the amplitudes of left-going and right-going solutions in the left lead (in layer 0 , for example) projected onto the $\nu$ th right-going and $\mu$ th left-going propagating states ( $\mathbf{k}$ vector real) and renormalized with the velocities so as to have unit flux. Determining these amplitudes requires finding the inverse of the Hamiltonian matrix of the scattering region modified by adding an embedding potential in order to incorporate the boundary conditions imposed by the leads. This matrix is finite and may be very sparse. Since only a few elements of its inverse are required, methods more efficient than direct matrix inversion can be used. The resulting numerical effort scales linearly with the extent of the scattering region in the transport direction.

\section{A. Muffin-tin orbitals and the KKR equation}

Muffin-tin orbitals ${ }^{40-43}$ (MTO) are defined for potentials in which space is divided into nonoverlapping, atomcentered "muffin-tin" spheres inside which the potential is spherically symmetric and the remaining "interstitial" region where the potential is taken to be constant. The atomic spheres approximation (ASA) is obtained (i) by taking the kinetic energy in the interstitial region to be zero and (ii) by expanding the muffin-tin spheres so that they fill all space whereby the volume of the interstitial region vanishes; for monoatomic solids such spheres are called atomic WignerSeitz (WS) spheres. Inside a WS (or MT) sphere at $\mathbf{R}$, the solution of the radial Schrödinger equation regular at $\mathbf{R}$, $\phi_{R l}\left(\varepsilon, r_{R}\right)$ can be determined numerically for energy $\varepsilon$ and angular momentum $l$ resulting in the partial wave (normalized in the sphere)

$$
\phi_{R l m}\left(\varepsilon, \mathbf{r}_{R}\right) \equiv \phi_{R L}\left(\varepsilon, \mathbf{r}_{R}\right) \equiv \phi_{R l}\left(\varepsilon, r_{R}\right) Y_{l m}\left(\hat{\mathbf{r}}_{R}\right)
$$

where $\hat{\mathbf{r}}$ is a unit vector, $\mathbf{r}_{R} \equiv \mathbf{r}-\mathbf{R}$, and $r_{R} \equiv|\mathbf{r}-\mathbf{R}|$. A continuous and differentiable orbital is constructed by attaching to the partial wave at the sphere boundary $r_{R} \equiv s_{R}$ a "tail" consisting of an appropriate linear combination of the solutions of the Laplace equation,

and

$$
J_{R L}^{0}\left(\mathbf{r}_{R}\right) \equiv\left(r_{R} / \omega\right)^{l}[2(2 l+1)]^{-1} Y_{L}\left(\hat{\mathbf{r}}_{R}\right)
$$

$$
K_{R L}^{0}\left(\mathbf{r}_{R}\right) \equiv\left(r_{R} / \omega\right)^{-l-1} Y_{L}\left(\hat{\mathbf{r}}_{R}\right),
$$

which are respectively, regular at $\mathbf{R}$ and at infinity. $\omega$ is the average WS radius if the structure contains different atoms. In terms of the logarithmic derivative of $\phi_{l}(\varepsilon, r)$ at $r \equiv s$

$$
D_{l}(\varepsilon, s) \equiv \frac{s \phi_{l}^{\prime}(\varepsilon, s)}{\phi_{l}(\varepsilon, s)}
$$

$\left[\phi_{l}^{\prime}(\varepsilon, s)\right.$ is the radial derivative], the radial solutions are matched if for $r \geqslant s$,

$$
\begin{aligned}
\phi_{l}(\varepsilon, r)= & \frac{l-D_{l}}{2 l+1}\left(\frac{s}{\omega}\right)^{l+1} \phi_{l}(\varepsilon, s) \\
& \times\left[K_{l}^{0}(r)-2(2 l+1)\left(\frac{\omega}{s}\right)^{2 l+1}\left(\frac{D_{l}+l+1}{D_{l}-l}\right) J_{l}^{0}(r)\right],
\end{aligned}
$$

where we drop the explicit $\mathbf{R}$-dependence when this does not give rise to ambiguity. Equation (7) can be rewritten ${ }^{52}$ in terms of the potential function,

$$
P_{l}^{0}(\varepsilon)=2(2 l+1)\left(\frac{\omega}{s}\right)^{2 l+1} \frac{D_{l}(\varepsilon)+l+1}{D_{l}(\varepsilon)-l},
$$

$$
\begin{gathered}
\text { and normalization } N_{l}^{0}(\varepsilon)=\frac{(2 l+1)}{\left(l-D_{l}\right)}\left(\frac{\omega}{s}\right)^{l+1} \frac{1}{\phi_{l}(\varepsilon, s)} \text {, as } \\
N_{l}^{0}(\varepsilon) \phi_{l}(\varepsilon, r)=K_{l}^{0}(r)-P_{l}^{0}(\varepsilon) J_{l}^{0}(r) .
\end{gathered}
$$

By subtracting from the partial wave, both inside and outside the MT sphere, the $J_{R L}^{0}\left(\mathbf{r}_{R}\right)$ component which is irregular at infinity, a function is formed which is continuous, differentiable, and regular in all space, an energy-dependent muffintin orbital $\chi_{R L}^{0}\left(\varepsilon, \mathbf{r}_{R}\right)$ : 


$$
\begin{aligned}
\chi_{R L}^{0}\left(\varepsilon, \mathbf{r}_{R}\right) & =N_{R l}^{0}(\varepsilon) \phi_{R l}(\varepsilon, \mathbf{r})+P_{R l}^{0}(\varepsilon) J_{R L}^{0}\left(\mathbf{r}_{R}\right) & & r_{R} \leqslant s_{R} \\
& =K_{L}^{0}\left(\mathbf{r}_{R}\right) & & r_{R}>s_{R}
\end{aligned}
$$

The tail $K_{R L}^{0}\left(\mathbf{r}_{R}\right)$ has the desirable property that closed forms exist for expanding it around a different site $\mathbf{R}^{\prime}$ in terms of the regular solutions $J_{R^{\prime} L^{\prime}}^{0}\left(\mathbf{r}_{R^{\prime}}\right)$,

$$
K_{R L}^{0}\left(\mathbf{r}_{R}\right)=-\sum_{L^{\prime}} J_{R^{\prime} L^{\prime}}^{0}\left(\mathbf{r}_{R^{\prime}}\right) S_{R^{\prime} L^{\prime}, R L}^{0}
$$

The expansion coefficients $S_{R^{\prime} L^{\prime}, R L}^{0}$ form a so-called canonical structure constant matrix: they do not depend on the lattice constant, on the MT (or AS) potentials, or on energy. Because of the augmentation with $J_{R L}^{0}\left(\mathbf{r}_{R}\right)$, the resulting MTO is no longer a solution of the Schrödinger equation (SE) inside its own sphere R. When, however, a solution of the SE is sought in the form of a linear combination of MTOs centered on different sites,

$$
\Psi(\varepsilon, \mathbf{r})=\sum_{R, L} \chi_{R L}^{0}\left(\varepsilon, \mathbf{r}_{R}\right) C_{R L}^{0}
$$

then the partial wave solution is recovered if the augmenting term $J_{R L}^{0}\left(\mathbf{r}_{R}\right)$ on site $\mathbf{R}$ is cancelled by the tails of MTOs centered on all other sites $\mathbf{R}^{\prime} \neq \mathbf{R}$, expanded about $\mathbf{R}$. The condition for this to occur is the "tail-cancellation" condition:

$$
\sum_{R^{\prime}, L^{\prime}}\left[P_{R L}^{0}(\varepsilon) \delta_{R R^{\prime}} \delta_{L L^{\prime}}-S_{R L, R^{\prime} L^{\prime}}^{0}\right] C_{R^{\prime} L^{\prime}}^{0}=0 .
$$

All of the information about the structural geometry of the system under investigation is contained in the structure constant matrix $S_{R L, R^{\prime} L^{\prime}}^{0}$ while all of the information about the atomic species on site $\mathbf{R}$ needed to calculate the electronic structure (eigenvalues and eigenvectors) is contained in the potential functions $P_{R L}^{0}(\varepsilon)$. These are determined by solving the radial Schrödinger equation for the corresponding spherically symmetrical atomic sphere potential for energy $\varepsilon$ and angular momentum $l$.

A disadvantage of these "conventional" MTOs is their infinite range. However, there is a remarkably simple generalization of the MTOs which allows their range to be modified by introducing a set of "screening" constants $\alpha_{R l}$ (not to be confused with the lead eigenmode index) while the "tailcancellation" condition remains essentially unchanged:

$$
\sum_{R^{\prime}, L^{\prime}}\left[P_{R L}^{\alpha}(\varepsilon) \delta_{R R^{\prime}} \delta_{L L^{\prime}}-S_{R L, R^{\prime} L^{\prime}}^{\alpha}\right] C_{R^{\prime} L^{\prime}}^{\alpha}=0
$$

$P^{\alpha}(\varepsilon)$ is a diagonal matrix related to $P^{0}(\varepsilon)$ by

$P^{\alpha}(\varepsilon)=P^{0}(\varepsilon)+P^{0}(\varepsilon) \alpha P^{0}(\varepsilon)=P^{0}(\varepsilon)\left[1-\alpha P^{0}(\varepsilon)\right]^{-1}$,

and

$$
S^{\alpha}=S^{0}+S^{0} \alpha S^{\alpha}=S^{0}\left(1-\alpha S^{0}\right)^{-1} .
$$

For any set of $\alpha_{R l}$, the energy-dependent MTOs with the normalization

$$
\sum_{R, L} \frac{\omega}{2} \dot{P}_{R L}^{\alpha}(\varepsilon)\left|C_{R L}^{\alpha}\right|^{2}=1
$$

form a complete set for the MT (AS) potential used in their construction. Here, $\dot{P}$ denotes an energy derivative and Eq. (18) follows from the relation $N^{\alpha}(\varepsilon)=\left[(\omega / 2) \dot{P}^{\alpha}(\varepsilon)\right]^{1 / 2}$. Sets of parameters $\alpha_{R l}$ have been found for which the "screened" structure constants $S_{R L, R^{\prime} L^{\prime}}^{\alpha}$ have very short range, decaying exponentially with the interatomic separation. ${ }^{41}$ The set of parameters, $\beta_{R l}$, which yields the shortest range MTOs is called the "tight-binding" (TB) representation. ${ }^{40}$ For closepacked structures, the range of $S_{R L, R^{\prime} L^{\prime}}^{\beta}$ is in practice limited to first- and second-nearest neighbors. This TB set, with $\alpha$ $=\beta$, is what we will use from now, unless stated otherwise, since it will allow us to define principal layers with a minimal thickness.

For the determination of energy bands $\varepsilon(\mathbf{k})$, the tailcancellation or KKR equations are inconvenient because the energy-dependence of the potential function makes it necessary to solve Eq. (14) or Eq. (15) by searching for the roots of a determinant, which is time consuming. Much more efficient methods have been developed based on energyindependent MTOs. However, to study transport we only need to know $P^{\beta}(\varepsilon)$ for a fixed energy, usually the Fermi energy. We assume that the Kohn-Sham equations have already been solved self-consistently (using, for example, a linearized method) so we have the potentials from which to calculate the potential functions. Although Eq. (15) can be brought into Hamiltonian form by linearizing the energy dependent potential function (see Appendix A), we will work directly with the more exact KKR equation.

\section{B. Eigenmodes of the leads}

We will assume that there exists two-dimensional translational symmetry in the plane perpendicular to the transport direction so that states can be characterized by a lateral wave vector $\mathbf{k}_{\|}$in the corresponding two-dimensional Brillouin zone. The screened KKR equation ${ }^{41}$ in the mixed representation of $\mathbf{k}_{\|}$and real space layer index $I$ (see Fig. 1) is

$$
-S_{I, I-1}^{\mathbf{k}_{\|}} \mathbf{C}_{I-1}+\left(P_{I, I}(\varepsilon)-S_{I, I}^{\mathbf{k}_{\|}}\right) \mathbf{C}_{I}-S_{I, I+1}^{\mathbf{k}_{\|}} \mathbf{C}_{I+1}=0,
$$

where $\mathbf{C}_{I} \equiv C_{I i} \equiv C_{I R l m}$ is a $\left(l_{\max }+1\right)^{2} H \equiv M$ dimensional vector describing the amplitudes of the $I$ th layer with $H$ sites and $\left(l_{\max }+1\right)^{2}$ orbitals per site. $P_{I, I}$ and $S_{I, J}$ are $M \times M$ matrices. $P_{I, I}$ is a diagonal matrix of potential functions characterizing the AS potentials of layer $I$ and

$$
S_{I, J}^{\mathbf{k}_{\|}}=\sum_{\mathbf{T} \in\left\{\mathbf{T}_{I, J}\right\}} S^{\beta}(\mathbf{T}) e^{i \mathbf{k}_{\|} \cdot \mathbf{T}},
$$

where $\left\{\mathbf{T}_{I, J}\right\}$ denotes the appropriate set of vectors that connect one lattice site in the Ith layer with lattice sites in the Jth layer.

By analogy with Eq. (2), Eq. (19) is the equation of motion we will use to calculate the amplitudes of right- and left-going waves which determine the scattering matrix. We 
will solve it for a fixed value of $\varepsilon$ (usually $\varepsilon_{F}$ ) and some $\mathbf{k}_{\|}$to find $k_{\mu}\left(\varepsilon, \mathbf{k}_{\|}\right)$the component of the Bloch wave vector in the transport direction. To keep the notation simple, explicit reference to the $\mathbf{k}_{\|}$and $\varepsilon$ dependence will be omitted from now on. The formalism to be described in the following can be applied to any electronic structure code based on the KKR equation (19), such as third-generation TB-LMTO. ${ }^{53-55}$

Let us first consider the Bloch states in the ideal lead. To obtain linearly independent solutions, we set $\mathbf{C}_{I}=\lambda^{I} \mathbf{C}_{0}$, since in a periodic potential the wave function should satisfy Bloch's theorem. The potential function matrix is the same for all unit cells. The structure constant matrix depends only on the relative positions and, because that is how they are defined, there is only coupling between adjacent principal layers so the equation of motion becomes

$$
\left(\begin{array}{cc}
S_{0,1}^{-1}\left(P_{0,0}-S_{0,0}\right) & -S_{0,1}^{-1} S_{1,0} \\
1 & 0
\end{array}\right)\left(\begin{array}{l}
\mathbf{C}_{I} \\
\mathbf{C}_{I-1}
\end{array}\right)=\lambda\left(\begin{array}{l}
\mathbf{C}_{I} \\
\mathbf{C}_{I-1}
\end{array}\right),
$$

The eigenvalue $\lambda_{\mu}$ can be written in the form $\lambda_{\mu}$ $=\exp \left(i \mathbf{k}_{\mu} \cdot \mathbf{T}_{0}\right)$ with $\mathbf{T}_{0}$ connecting equivalent sites in adjacent principal layers. The wave vector $\mathbf{k}_{\mu}$ can be decomposed into $\mathbf{k}_{\|}$and a remainder which is in general not real, $\mathbf{k}_{\mu}=\left(\mathbf{k}_{\|}, \mathbf{k}_{\mu}-\mathbf{k}_{\|}\right)$. Equation (21) has $2 M$ eigenvalues and $2 M$ eigenvectors, corresponding to $M$ right-going and $M$ leftgoing waves. By calculating the wave vectors and velocities [see Eq. (38) and Appendix A] of the lead eigenmodes, the propagating and evanescent states can be identified and sorted into right-going or left-going modes.

Letting $\mathbf{u}_{1}(-), \ldots, \mathbf{u}_{M}(-)$ denote the left-going solutions $\mathbf{C}_{0}$ corresponding to eigenvalues $\lambda_{1}(-), \ldots, \lambda_{M}(-)$ and $\mathbf{u}_{1}(+), \ldots, \mathbf{u}_{M}(+)$ the right-going solutions corresponding to eigenvalues $\lambda_{1}(+), \ldots, \lambda_{M}(+)$, the matrix $U_{i \mu}( \pm)$ is defined as

$$
U( \pm)=\left[\mathbf{u}_{1}( \pm) \cdots \mathbf{u}_{M}( \pm)\right]
$$

and the matrix $\Lambda( \pm)$ as the diagonal matrix with elements $\lambda_{\mu}( \pm)$. Following Ando, we next expand any left- or rightgoing wave, at $I=0$, for example, as

$$
\mathbf{C}_{0}( \pm)=U( \pm) \mathbf{C}( \pm) .
$$

Note that $\mathbf{C}_{0}$ is a vector whose elements are labeled $i$ while the elements of the vector $\mathbf{C}$ are labeled $\mu$.

$$
F( \pm) \equiv U( \pm) \Lambda_{ \pm} U^{-1}( \pm)
$$

is the matrix of Bloch factors (including evanescent states) transformed onto the basis $|i\rangle$ and plays a central role in the following. Knowing it makes it possible to translate a state expressed in the basis $|i\rangle$ from layer $J$ of the lead to layer $I$ by

$$
\mathbf{C}_{I}( \pm)=F^{I-J}( \pm) \mathbf{C}_{J}( \pm) .
$$

The $F$ matrices can be used to find the Green functions of the lead (but it should be emphasized that these are not required in Ando's approach). For example, the retarded surface Green function of the semi-infinite lead extending from $i=-\infty \cdots 0$ is given simply by

$$
g(\varepsilon)=F^{-1}(-) S_{01}^{-1} .
$$

The procedure described in this section should therefore be seen as an alternative to the recursive schemes for obtaining the surface Green functions commonly found in the literature (see, e.g., Ref. 44). The reader is referred to Refs. 56 and 57 for more detailed discussion.

\section{Scattering problem}

The scattering region $\mathcal{S}$, divided into $N$ principal layers numbered 1 to $N$, is now inserted between the left and right leads. The resulting (scattering region+leads) problem is infinite dimensional in the real space MTO representation but, by making use of their translational symmetry, the leads can be incorporated as boundary conditions and the scattering problem can be reduced to a finite problem whose dimension is determined by the size of the scattering region (number of sites $\times$ number of orbitals per site).

We set about decoupling the scattering region from the leads, first on the left-hand side, then on the right. The amplitude in the zeroth layer is first separated into right- and left-going components $\mathbf{C}_{0}=\mathbf{C}_{0}(+)+\mathbf{C}_{0}(-)$. Because there is no scattering of Bloch states in the leads, the right- and leftgoing components can be translated to the left by one (principal layer) lattice spacing using the generalized Bloch factors (25) so the amplitude in layer -1 can be related to that in layer 0 as

$$
\begin{aligned}
\mathbf{C}_{-1} & =F_{\mathcal{L}}^{-1}(+) \mathbf{C}_{0}(+)+F_{\mathcal{L}}^{-1}(-) \mathbf{C}_{0}(-) \\
& =\left[F_{\mathcal{L}}^{-1}(+)-F_{\mathcal{L}}^{-1}(-)\right] \mathbf{C}_{0}(+)+F_{\mathcal{L}}^{-1}(-) \mathbf{C}_{0},
\end{aligned}
$$

allowing us to express $\mathbf{C}_{-1}$ in terms of $\mathbf{C}_{0}$ and $\mathbf{C}_{0}(+)$ and so eliminate it from the equation of motion for the zeroth layer

$$
-S_{0,-1} \mathbf{C}_{-1}+\left(P_{0,0}-S_{0,0}\right) \mathbf{C}_{0}-S_{0,1} \mathbf{C}_{1}=0,
$$

which becomes

$$
\left(P_{0,0}-\widetilde{S}_{0,0}\right) \mathbf{C}_{0}-S_{0,1} \mathbf{C}_{1}=S_{0,-1}\left[F_{\mathcal{L}}^{-1}(+)-F_{\mathcal{L}}^{-1}(-)\right] \mathbf{C}_{0}(+) .
$$

Here $\mathcal{L}$ denotes the left lead and $\widetilde{S}_{0,0}=S_{0,0}+S_{0,-1} F_{\mathcal{L}}^{-1}(-)$. The quantity $S_{0,-1} F_{\mathcal{L}}^{-1}(-)$ is the "embedding potential" for the left lead and the net result is that the equations of motion have been truncated at layer 0 .

On the right-hand side of the scattering region, we are interested in the situation where only right-going waves can exist in the $(N+1)$ th layer, so

$$
\mathbf{C}_{N+2}=F_{\mathcal{R}}(+) \mathbf{C}_{N+1}(+)
$$

allowing $\mathbf{C}_{N+2}$ to be eliminated from the EOM for $\mathbf{C}_{N+1}$

$$
\left(P_{N+1, N+1}-\widetilde{S}_{N+1, N+1}\right) \mathbf{C}_{N+1}-S_{N+1, N} \mathbf{C}_{N}=0,
$$

where $\tilde{S}_{N+1, N+1}=S_{N+1, N+1}+S_{N+1, N+2} F_{\mathcal{R}}(+)$ and $S_{N+1, N+2} F_{\mathcal{R}}(+)$ is the embedding potential for the right lead.

Making use of the lead boundary conditions, the tail cancellation condition for the scattering problem in real space is given by the set of inhomogeneous linear equations 


$$
\left(\begin{array}{cccccc}
(P-\widetilde{S})_{0,0} & -S_{0,1} & 0 & \cdots & 0 & 0 \\
-S_{1,0} & (P-S)_{1,1} & -S_{1,2} & \cdots & 0 & 0 \\
0 & -S_{2,1} & (P-S)_{2,2} & \cdots & \vdots & 0 \\
\vdots & \vdots & \cdots & \ddots & \vdots & 0 \\
0 & 0 & \cdots & \cdots & (P-S)_{N, N} & -S_{N, N+1} \\
0 & 0 & 0 & \cdots & -S_{N+1, N} & (P-\widetilde{S})_{N+1, N+1}
\end{array}\right)\left(\begin{array}{l}
\mathbf{C}_{0} \\
\mathbf{C}_{1} \\
\mathbf{C}_{2} \\
\vdots \\
\mathbf{C}_{N} \\
\mathbf{C}_{N+1}
\end{array}\right) \equiv(\mathbf{P}-\widetilde{\mathbf{S}})\left(\begin{array}{l}
\mathbf{C}_{0} \\
\mathbf{C}_{1} \\
\mathbf{C}_{2} \\
\vdots \\
\mathbf{C}_{N} \\
\mathbf{C}_{N+1}
\end{array}\right)
$$

$$
=\left(\begin{array}{c}
S_{0,-1}\left[F_{\mathcal{L}}^{-1}(+)-F_{\mathcal{L}}^{-1}(-)\right] \mathbf{C}_{0}(+) \\
0 \\
0 \\
\vdots \\
0 \\
0
\end{array}\right)
$$

which can be solved in terms of $\mathbf{g}=(\mathbf{P}-\widetilde{\mathbf{S}})^{-1}$

$$
\left(\begin{array}{l}
\mathbf{C}_{0} \\
\mathbf{C}_{1} \\
\mathbf{C}_{2} \\
\vdots \\
\mathbf{C}_{N} \\
\mathbf{C}_{N+1}
\end{array}\right)=\mathbf{g}\left(\begin{array}{c}
S_{0,-1}\left[F_{\mathcal{L}}^{-1}(+)-F_{\mathcal{L}}^{-1}(-)\right] \mathbf{C}_{0}(+) \\
0 \\
0 \\
\vdots \\
0 \\
0
\end{array}\right)
$$

This treatment is very similar to the widely used surface Green function method. ${ }^{56}$ The boundary conditions in Eq. (32) are explicitly defined by considering the Bloch wave coming from the left-hand side while for conventional retarded or advanced Green functions the boundary conditions are specified by an infinitesimal imaginary part of the energy parameter $\varepsilon$.

We are now in a position where we can relate the outgoing wave amplitude in the right electrode to the incoming wave in the left electrode through the Green function by

$$
\mathbf{C}_{N+1}(+)=\mathbf{C}_{N+1}=g_{N+1,0} S_{0,-1}\left[F_{\mathcal{L}}^{-1}(+)-F_{\mathcal{L}}^{-1}(-)\right] \mathbf{C}_{0}(+) .
$$

Using the transformation between the eigenstates and the localized basis functions $U_{i \alpha}( \pm)$, we obtain the transmission and reflection matrix elements ${ }^{45}$

$$
\begin{gathered}
t_{\mu \nu}=\left(\frac{v_{\mu}}{v_{\nu}}\right)^{1 / 2}\left\{U_{\mathcal{R}}^{-1}(+) g_{N+1,0} S_{0,-1}\left[F_{\mathcal{L}}^{-1}(+)-F_{\mathcal{L}}^{-1}(-)\right] U_{\mathcal{L}}(+)\right\}_{\mu \nu}, \\
r_{\mu \nu}=\left(\frac{v_{\mu}}{v_{\nu}}\right)^{1 / 2}\left\{U_{\mathcal{L}}^{-1}(-)\left\langle g_{0,0} S_{0,-1}\left[F_{\mathcal{L}}^{-1}(+)-F_{\mathcal{L}}^{-1}(-)\right]-1\right\rangle U_{\mathcal{L}}(+)\right\}_{\mu \nu},
\end{gathered}
$$

where $\mu$ and $\nu$ label Bloch states and $v_{\mu}, v_{\nu}$ are the components of the corresponding group velocities in the transport direction. Similarly, an incident wave from the right side is transmitted or reflected as

$$
\begin{gathered}
t_{\mu \nu}^{\prime}=\left(\frac{v_{\mu}}{v_{\nu}}\right)^{1 / 2}\left\{U_{\mathcal{L}}^{-1}(-) g_{0, N+1} S_{N+1, N+2}\left[F_{\mathcal{R}}(-)-F_{\mathcal{R}}(+)\right] U_{\mathcal{R}}(-)\right\}_{\mu \nu}, \\
r_{\mu \nu}^{\prime}=\left(\frac{v_{\mu}}{v_{\nu}}\right)^{1 / 2}\left\{U_{\mathcal{R}}^{-1}(+)\left\langle g_{N+1, N+1} S_{N+1, N+2}\left[F_{\mathcal{R}}(-)-F_{\mathcal{R}}(+)\right]-1\right\rangle U_{\mathcal{R}}(-)\right\}_{\mu \nu} .
\end{gathered}
$$


The group velocities in Eqs. (34)-(37) are determined using the expression

$$
v_{\mu}( \pm)=\frac{i d}{\hbar}\left[\mathbf{u}_{\mu}^{\dagger}( \pm) S_{I, I+1}^{\mathbf{k}_{\|}} \mathbf{u}_{\mu}( \pm) \lambda_{\mu}-\text { H.c. }\right]
$$

which is derived in Appendix A. Here, $I$ and $I+1$ denote neighboring principal layers in either left or right lead, $d$ $=\mathbf{T}_{0} \cdot \hat{n}$ is the distance between equivalent monolayers in adjacent principal layers, and $\hat{n}$ is a unit vector in the transport direction.

The conductance can now be calculated using the elements of the scattering matrix required in the LandauerBüttiker formula (1), the transmission matrix (34). It can be shown $^{56}$ that this is fully equivalent to the popular Caroli's nonequilibrium Green functions formula. ${ }^{58}$ It is, however, expressed in a more physically transparent fashion and avoids the use of complex energies in the Green's function approach (where they were introduced to stabilize the calculation of the surface Green function and to make selfconsistent iteration more efficient by expressing the density matrix as a contour integral).

\section{Disorder}

Interfaces between materials with different lattice parameters $^{48}$ and disordered interfaces ${ }^{9,49}$ can be modeled very flexibly using lateral supercells. This approach allows us to study the effect of various types of disorder on transport properties, ranging from homogeneous interdiffusion (alloying) to islands, steps, etc. The supercell description of disorder becomes formally exact in the limit of infinitely large supercells. In practice, satisfactory convergence is achieved for supercells of quite moderate size (see Sec. III C).

\section{Leads}

The factor limiting the "size" of the scattering problem which can be handled in practice is the rank of the blocks of the block-tridiagonal equation of motion (19), which is proportional to the number of atoms in the lateral supercell. If performed straightforwardly in the manner outlined in Sec. II B, the solution of the lead equation (21) involves solving a non-Hermitian eigenvalue problem whose rank is twice as large. Unless use is made of the greater translational symmetry present in the leads, this can become the limiting step in the whole calculation. Doing so makes it possible to reduce the dimension of the lead state calculation to a size determined by the dimension of a primitive unit cell which is usually negligible.

We consider an $H_{1} \times H_{2}$ lateral supercell defined by the real-space lattice vectors

$$
\mathbf{A}_{1}=H_{1} \mathbf{a}_{1} \text { and } \mathbf{A}_{2}=H_{2} \mathbf{a}_{2}
$$

where $\mathbf{a}_{1}$ and $\mathbf{a}_{2}$ are the lattice vectors describing the in-plane periodicity of a primitive unit cell (Fig. 2). The cells contained within the supercell are generated by the set of translations
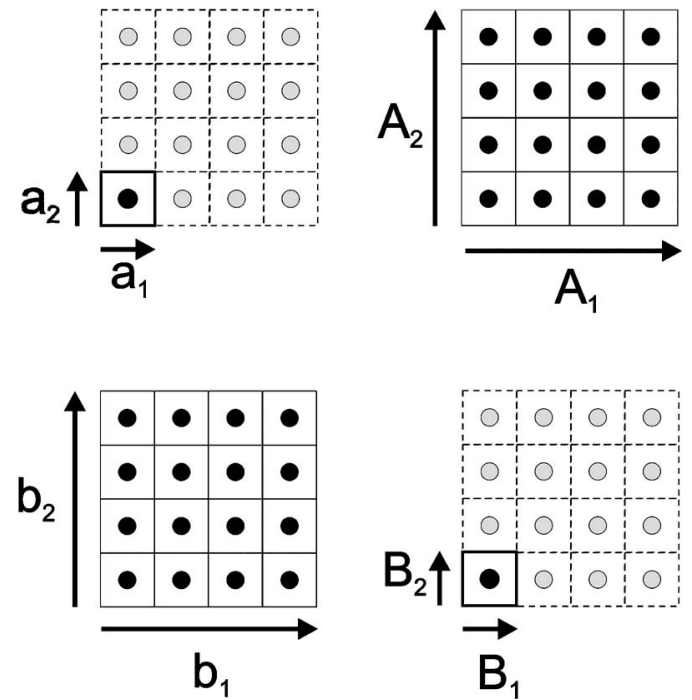

FIG. 2. Illustration of lateral supercells and corresponding 2D interface Brillouin zones. Top panel: lattice vectors for a primitive unit cell containing a single atom [left-hand side (lhs)] and a 4 $\times 4$ supercell [right-hand side (rhs)]. Bottom panel: a single $k$-point in the $\mathrm{BZ}$ (rhs) corresponding to the $4 \times 4$ real-space supercell is equivalent to $4 \times 4 k$-points in the $\mathrm{BZ}$ (lhs) corresponding to the real-space primitive unit cell.

$$
\begin{aligned}
\mathbf{T}_{\|_{\mathcal{T}}} \in \mathbb{T}= & \left\{\mathbf{T}_{\|}=h_{1} \mathbf{a}_{1}+h_{2} \mathbf{a}_{2} ;\right. \\
& \left.0 \leqslant h_{1}<H_{1}, 0 \leqslant h_{2}<H_{2}\right\},
\end{aligned}
$$

where $\mathcal{T}=1, \ldots, H_{1} \times H_{2}$ is a convenient cell index. In reciprocal space the supercell Brillouin zone is defined by the reduced vectors

$$
\mathbf{B}_{1}=\mathbf{b}_{1} / H_{1} \text { and } \mathbf{B}_{2}=\mathbf{b}_{2} / H_{2}
$$

where $\mathbf{b}_{1}$ and $\mathbf{b}_{2}$ are the reciprocal lattice vectors corresponding to the real space primitive unit cell. As a result the Brillouin zone (BZ) is folded down, as shown schematically in Fig. 2 (bottom rhs), and the single $\mathbf{k}_{\|}^{\mathrm{S}}$ point ( $\mathrm{S}$ is used to label supercell quantities) in the supercell $\mathrm{BZ}$ corresponds to the set of $H_{1} \times H_{2} \mathbf{k}_{\|}$points in the original unfolded BZ

$$
\begin{aligned}
\mathbf{k}_{\|_{\mathcal{K}}} \in \mathbb{K}= & \left\{\mathbf{k}_{\|}=\mathbf{k}_{\|}^{\mathrm{S}}+h_{1} \mathbf{B}_{1}+h_{2} \mathbf{B}_{2} ;\right. \\
& \left.0 \leqslant h_{1}<H_{1}, 0 \leqslant h_{2}<H_{2}\right\}
\end{aligned}
$$

with $\mathcal{K}=1, \ldots, H_{1} \times H_{2}$. Solutions associated with different $\mathbf{k}_{\| \mathcal{K}}$ in the primitive unit cell representation become different "bands" at the single $\mathbf{k}_{\|}^{\mathrm{S}}$ in the supercell representaton.

The indices $\mathcal{T}$ and $\mathcal{K}$ provide a natural means of describing the supercell-related matrices $U^{\mathrm{S}}( \pm)$ and $F^{\mathrm{S}}( \pm)$ and their inverses in terms of $\left(H_{1} \times H_{2}\right)^{2}$ sub-blocks with dimensions defined by the primitive unit cell. Thus $U_{\mathcal{T} \mathcal{S}}^{\mathrm{S}}( \pm)$ is the block containing the amplitudes of the modes associated with $\mathbf{k}_{\|_{\mathcal{K}}}$ in the $\mathcal{T}$ th real-space cell.

Solving the single unit cell problem for the set of $\mathbf{k}_{\|-}$-points belonging to $\mathbb{K}$ (lhs of Fig. 2) and using the Bloch symmetry of the eigenmodes, we get trivially 


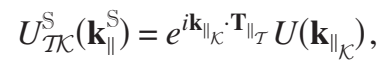

where $U\left(\mathbf{k}_{\|_{\mathcal{K}}}\right)$ is the matrix (22) of modes for a primitive unit cell for $\mathbf{k}_{\|_{\mathcal{K}}}$ and the \pm qualifier has been dropped for simplicity. Defining the matrix of phase factors

$$
X\left(\mathbf{k}_{\|}^{\mathrm{S}}\right)=\left(\begin{array}{ccc}
e^{i \mathbf{k} \|_{1}} \cdot \mathbf{T}_{\|_{1}} & \ldots & e^{i \mathbf{k} \|_{H} \cdot \mathbf{T}_{\|_{1}}} \\
\vdots & & \vdots \\
e^{i \mathbf{i} \|_{1} \cdot \mathbf{T}_{\|_{H}}} & \ldots & e^{i \mathbf{k}_{H} \cdot \mathbf{T}_{\|_{H}}}
\end{array}\right)
$$

with $H \equiv H_{1} \times H_{2}$, and its inverse $Y=X^{-1}$, we can straightforwardly determine

$$
\left[U^{\mathrm{S}}\left(\mathbf{k}_{\|}^{\mathrm{S}}\right)\right]_{\mathcal{K} \mathcal{T}}^{-1}=U^{-1}\left(\mathbf{k}_{\|_{\mathcal{K}}}\right) Y_{\mathcal{K} \mathcal{T}}
$$

and

$$
F_{\mathcal{T}_{1} \mathcal{T}_{2}}^{\mathrm{S}}\left(\mathbf{k}_{\|}^{\mathrm{S}}\right)=\sum_{\mathcal{K}} X_{\mathcal{T}_{1} \mathcal{K}} F\left(\mathbf{k}_{\|}\right) Y_{\mathcal{K} \mathcal{T}_{2}} .
$$

The procedure outlined above for determining the matrices describing the lead modes scales linearly with the size of the supercell, i.e., as $\left(H_{1} \times H_{2}\right)$ rather than as $\left(H_{1} \times H_{2}\right)^{3}$ which is the scaling typical for matrix operations. Another advantage is that it enables us to analyze the scattering. By keeping track of the relation between supercell "bands" and equivalent eigenmodes at different $\mathbf{k}_{\|_{\mathcal{K}}}$ (Fig. 2) we can straightforwardly obtain from Eqs. (34)-(37) $t_{\mu \nu}\left(\mathbf{k}_{\|_{\mathcal{K}_{1}}}, \mathbf{k}_{\|_{\mathcal{K}_{2}}}\right)$ and other scattering coefficients. In other words the "interband" specular scattering in the supercell picture translates, in the presence of disorder in the scattering region, into the "diffuse" scattering between the $\mathbf{k}_{\|}$vectors belonging to the K set.

\section{CALCUlations}

Even though the theoretical scheme outlined above contains no adjustable parameters, its practical implementation does involve numerous approximations, some physical, others numerical, which need to be evaluated. At present, any workable scheme must be based upon an independent particle approximation. The results of a transport calculation will be limited by the extent to which the single particle electronic structures used are consistent with the corresponding Fermi surfaces determined experimentally using methods such as de Haas-van Alphen measurements or the occupied and unoccupied electronic states close to the Fermi energy determined by, for example, photoelectron spectroscopy.

In this section we examine how various approximations affect our end results. We begin with the calculation of the scattering states in bulk $\mathrm{Cu}$ and bulk Co (III A). These are then used to study specular scattering from an ideal ordered $\mathrm{Cu} / \mathrm{Co}(111)$ interface (III B) after which we describe how we model disordered interfaces (III C) and how the results can be analyzed (IIID), and are related to experiment (IIIE).

\section{A. Leads}

For a crystalline conductor with Bloch translational symmetry, each state at the Fermi energy can move unhindered through the solid so that the transmission matrix is diagonal

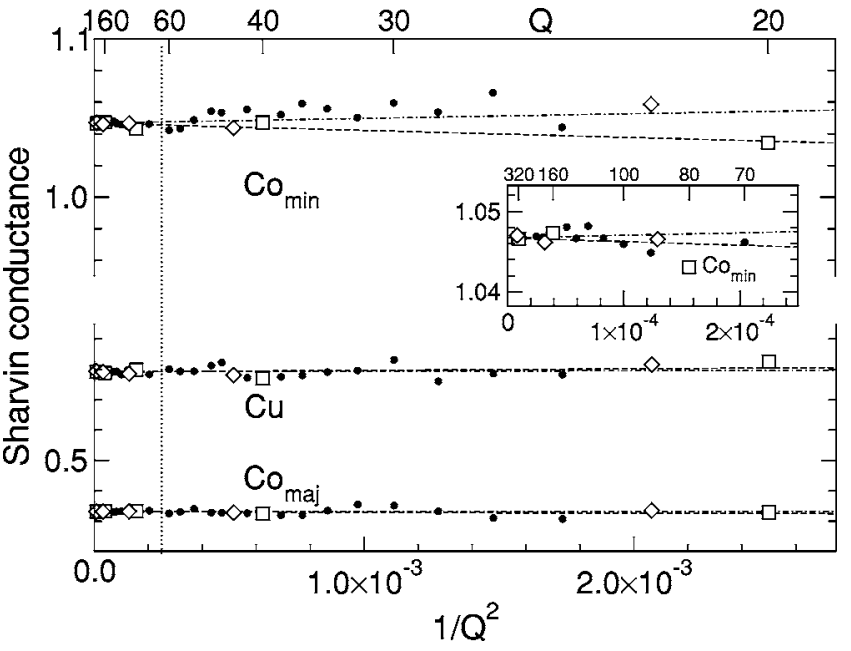

FIG. 3. Sharvin conductance $G^{\sigma}(111)$ (in units of $10^{15} \Omega^{-1} \mathrm{~m}^{-2}$ ) for bulk fcc $\mathrm{Cu}$ and $\mathrm{Co}$ (majority and minority spin) plotted as a function of the normalized area element used in the Brillouin zone summation, $\Delta^{2} \mathbf{k}_{\|} / A_{B Z}=1 / Q^{2}$. $Q$, the number of intervals along the reciprocal lattice vector is indicated at the top of the figure. The dashed line is the weighted (weighting $Q^{2}$ ) least-squares fit to the series $Q=20,40,80,160,320$ shown as squares; the dash-dotted line is the weighted least-squares fit to the series $Q$ $=22,44,88,176,352$ shown as diamonds. The part of the curve for the Co minority spin case to the left of the vertical dotted line is shown on an expanded scale in the inset. An fcc lattice constant of $a=3.614 \AA$ and $s p d$ basis were used together with von BarthHedin's exchange-correlation potential.

with $\left|t_{\mu \nu}\right|^{2}=\delta_{\mu \nu}$. In this ballistic regime, Eq. (1) reduces to

$$
G^{\sigma}(\hat{n})=\frac{e^{2}}{h} \sum_{\mu \mathbf{k}_{\|}}\left|t_{\mu \mu}^{\sigma}\left(\mathbf{k}_{\|}\right)\right|^{2}=\frac{e^{2}}{h} N^{\sigma}(\hat{n})
$$

and calculation of the so-called Sharvin conductance becomes a matter of counting the number of modes (channels) propagating in the transport direction $\hat{n}$, denoted in Eq. (47) as $N^{\sigma}(\hat{n})$. To solve Eq. (21) in practice, the orbital angular momentum expansions in Eqs. (12) and (13), which are in principle infinite, must be truncated by introducing some cutoff in $l$, denoted $l_{\max }$. Usually, a value of $l_{\max }=2$ or 3 is used, corresponding to $s p d$ - or $s p d f$-bases.

The $\mathbf{k}_{\|}$summation is carried out by sampling, on a regular mesh, the 2D Brillouin zone (BZ) defined by the (lateral) translational periodicity perpendicular to $\hat{n}$. The results of carrying out this BZ summation are shown in Fig. 3 where $G^{\sigma}(\hat{n})$ is plotted as a function of $\Delta^{2} k_{\|} / A_{B Z}$, the normalized area element per $\mathbf{k}_{\|}$-point for bulk fcc $\mathrm{Cu}$ and for the majority and minority spins of bulk fcc Co. When the 2D-BZ reciprocal lattice vectors are each divided into $Q$ intervals, then $\Delta^{2} k_{\|} / A_{B Z}=1 / Q^{2}$. It can be seen that the Sharvin conductance is converged to about $1 \%$ if $3600=60 \times 60$ points are used in the complete 2D-BZ and to about $0.2 \%$ for $102400=320 \times 320$ sampling points. The worst case is for the minority spin of Co which has a complex multisheeted Fermi surface. To see if there are any simple underlying trends in the convergence, we repeatedly bisect the intervals used in the BZ summation starting with $Q=20$ and $Q=22$, 
shown in the figure as squares and diamonds, respectively, and least-squares fitted with the dashed and dash-dotted lines. The convergence is fairly uniform but not very systematic indicating that the summation is limited by fine structure in the integrand at the smallest length scale studied which can only be resolved by increasingly fine sampling. Thus there is nothing to be gained by developing more sophisticated interpolation schemes and when we introduce disorder in Sec. III C, this will be even more so. However, in the following we will see that the level of convergence we can achieve with discrete sampling is quite adequate and not a limiting step in the whole procedure.

The calculations shown in the figure were performed using an $s p d$-basis, for an fcc lattice constant $a=3.614 \AA$ corresponding to the experimental volume of bulk (fcc) $\mathrm{Cu}$ and using the exchange-correlation potential calculated and parameterized by von Barth and Hedin. ${ }^{59}$ For convenience, and to avoid repetition, we will refer to this in the following as a "standard" configuration. The converged values are given (underlined) in Table I together with values calculated using an fcc lattice constant $a=3.549 \AA$ corresponding to the volume of bulk hcp Co. ${ }^{60}$ Because we shall be studying $\mathrm{Cu} / \mathrm{Co}$ interfaces where the volume per atom is not known very precisely from experiment, we will want to estimate the variation that can be expected when different but equally reasonable lattice constants are used. The increase of 3.4\% (from 0.558 to $0.577 \times 10^{15} \Omega^{-1} \mathrm{~m}^{-2}$ ) observed for $\mathrm{Cu}$ can be attributed to the increased areal density of $\mathrm{Cu}$ atoms, $(3.614 / 3.549)^{2}$ corresponding to $\sim 3.7 \%$. The Table also contains the corresponding results obtained with an $s p d f$-basis. To the numerical accuracy shown, there is no difference between the $s p d$ and $s p d f$ case for $\mathrm{Cu}$.

For Co majority spin states, there is a $4 \%$ decrease in the conductance on going from an $s p d$ - to an $s p d f$-basis. For a lattice constant $a=3.614 \AA$, the magnetization is $1.684 \mu_{B} /$ atom for an $s p d$ - and $1.648 \mu_{B} /$ atom for an $s p d f$-basis corresponding, respectively, to $n_{\text {maj }}=5.342$ and 5.324 electrons in the majority spin bands. Since all five (nominal) majority-spin $d$ bands are full there are 0.342 and 0.324 electrons in the free-electron-like $s p$ band. In a free electron picture the ratio of the projection of the spherical Fermi surfaces is $(0.324 / 0.342)^{2 / 3}=0.96$, thus explaining the observed numerical result.

The Co majority-spin conductance scarcely changes with changing lattice constant, however. The origin of this behavior lies in the volume dependence of the magnetic moment. When the lattice constant is decreased, the $d$ bands broaden and the magnetic moment decreases from 1.684 to $1.646 \mu_{B} /$ atom in the $s p d$ case with a corresponding decrease of the occupancy of the $s p$ band from 0.342 to 0.323 majority-spin electrons. The corresponding $4 \%$ decrease in conductance is almost perfectly compensated by the increased areal density of atoms so there is no net change. For the minority-spin conductance, the same factors play a role but now the $d$ bands are only partly filled. This results in complex Fermi surfaces for which simple estimates cannot be made. In this case recourse must be made to full band structure calculations. We return to this in Sec. III B.

The calculations presented so far were carried out using the exchange-correlation potential calculated and parameter-
TABLE I. The Sharvin conductances per spin (in units of $10^{15} \Omega^{-1} \mathrm{~m}^{-2}$ ) in the (111) direction for fcc $\mathrm{Cu}$ and $\mathrm{Co}$ using the experimental volumes of $\mathrm{Cu}$ and $\mathrm{Co}$. The underlined numbers are the converged values discussed in relation to Fig. 3. Most of the results were obtained with von Barth-Hedin's exchange-correlation potential while the results in brackets are for Perdew-Zunger (PZ) and Vosko-Wilk-Nusair (VWN) parameterizations, respectively. Where a single number is given in brackets, it means that $\mathrm{PZ}$ and VWN potentials yield identical results to the accuracy given. The corresponding results of Schep et al. are given in the last column. The number of electrons with spin $\sigma$ is given in the fourth column.

\begin{tabular}{|c|c|c|c|c|c|}
\hline & \multirow[b]{2}{*}{$a(\AA)$} & \multirow[b]{2}{*}{ Basis } & \multirow[b]{2}{*}{$n_{\sigma}$} & \multicolumn{2}{|l|}{$G^{\sigma}(111)$} \\
\hline & & & & Present calculation & Schep $^{a}$ \\
\hline \multirow[t]{4}{*}{ Copper } & 3.549 & spd & 5.5 & $0.577(0.577,0.577)$ & 0.57 \\
\hline & 3.549 & spdf & 5.5 & $0.577(0.577)$ & \\
\hline & 3.614 & spd & 5.5 & $\underline{0.558}(0.559)$ & 0.55 \\
\hline & 3.614 & spdf & 5.5 & $0.558(0.558)$ & 0.55 \\
\hline \multirow{4}{*}{$\begin{array}{l}\text { Cobalt } \\
\text { majority }\end{array}$} & 3.549 & spd & 5.323 & $0.469(0.459,0.467)$ & 0.45 \\
\hline & 3.549 & $\operatorname{spdf}$ & 5.304 & $0.449(0.440)$ & 0.43 \\
\hline & 3.614 & spd & 5.342 & $\underline{0.466}(0.457)$ & 0.45 \\
\hline & 3.614 & spdf & 5.324 & $0.448(0.439)$ & \\
\hline \multirow{4}{*}{$\begin{array}{l}\text { Cobalt } \\
\text { minority }\end{array}$} & 3.549 & spd & 3.677 & $1.082(1.081,1.082)$ & 1.10 \\
\hline & 3.549 & $s p d f$ & 3.696 & $1.120(1.125)$ & 1.13 \\
\hline & 3.614 & spd & 3.658 & $\underline{1.046}(1.047)$ & 1.06 \\
\hline & 3.614 & $\operatorname{spdf}$ & 3.676 & $1.074(1.079)$ & \\
\hline
\end{tabular}

${ }^{\text {aReference } 37 .}$

ized by von Barth and Hedin. ${ }^{59}$ This is only one of a number of potentials we could have used, none of which is clearly better than the others in describing the ground state properties of magnetic materials. To gauge the uncertainty arising from this arbitrary choice, a number of calculations were carried out using the potentials given by Perdew-Zunger ${ }^{61}$ and Vosko-Wilk-Nusair ${ }^{62}$ and the results are given in brackets in the table. Using different exchange-correlation potentials leads to variation in the conductances of the order of $1 \%$ or $2 \%$.

A different (but equivalent) approach was adopted by Schep et al. ${ }^{1,37}$ to the determination of the Sharvin conductances for the same systems using conventional firstprinciples LMTO-ASA bulk electronic band structures, i.e., using $\varepsilon_{i}(\mathbf{k})$ rather than $k_{\mu}\left(\varepsilon=\varepsilon_{F}, \mathbf{k}_{\|}\right)$as used here. He expressed the Sharvin conductance as a projection of the Fermi surface onto a plane perpendicular to the transport direction and calculated the areas using a suitably modified 3D-BZ integration scheme. His results are also given in Table I and are as consistent with our present values as can be expected when using two entirely different computer codes.

In determining the conductance of the leads, the BZ summation does not present a problem. The uncertainties arising from small variations in the atomic volumes, from incompleteness of the basis and from the choice of LDA parameterization are of comparable size. The MTO-AS approximation can be systematically improved but only at substantial computational cost. Since there is currently no way to sys- 
TABLE II. Variation of the layer-resolved magnetic moments (in Bohr magnetons) for $\mathrm{Cu} / \mathrm{Co}(111)$ interfaces with basis set and lattice constant. The main results were obtained with von BarthHedin's exchange-correlation potential while the results in brackets, where given, are for Perdew-Zunger and Vosko-Wilk-Nusair parameterizations, respectively. The underlined conductances are the converged values discussed in relation to Fig. 4. In the last two rows, the interface conductances are given in units of $10^{15} \Omega^{-1} \mathrm{~m}^{-2}$.

\begin{tabular}{|c|c|c|c|c|}
\hline \multirow{2}{*}{$\begin{array}{l}a(\AA) \\
\text { Basis }\end{array}$} & \multicolumn{2}{|c|}{3.549} & \multirow{2}{*}{$\begin{array}{c}3.582 \\
\text { spd }\end{array}$} & \multirow{2}{*}{$\begin{array}{l}3.614 \\
\text { spd }\end{array}$} \\
\hline & $s p d f$ & spd & & \\
\hline$m_{\mathrm{Cu}}($ bulk $)$ & 0.000 & 0.000 & 0.000 & 0.000 \\
\hline$m_{\mathrm{Cu}}($ int -4$)$ & $0.001(1)$ & 0.001 & 0.001 & 0.001 \\
\hline$m_{\mathrm{Cu}}($ int -3$)$ & $-0.001(0)$ & 0.000 & 0.000 & 0.000 \\
\hline$m_{\mathrm{Cu}}($ int -2$)$ & $-0.005(5)$ & $-0.005(4,5)$ & $-0.005(4)$ & -0.005 \\
\hline$m_{\mathrm{Cu}}($ int -1$)$ & $0.002(4)$ & $0.004(6,4)$ & $0.003(4)$ & $0.001(2)$ \\
\hline$m_{\mathrm{Co}}(\mathrm{int}+1)$ & $1.526(490)$ & $1.578(45,73)$ & $1.605(573)$ & $1.636(01)$ \\
\hline$m_{\mathrm{Co}}(\mathrm{int}+2)$ & 1.621(597) & $1.656(35,53)$ & $1.673(53)$ & $1.690(70)$ \\
\hline$m_{\mathrm{Co}}(\mathrm{int}+3)$ & $1.602(576)$ & $1.645(21,41)$ & $1.662(39)$ & $1.680(59)$ \\
\hline$m_{\mathrm{Co}}(\mathrm{int}+4)$ & $1.610(587)$ & $1.649(27,45)$ & $1.665(45)$ & $1.683(62)$ \\
\hline$m_{\mathrm{Co}}($ bulk $)$ & $1.609(590)$ & $1.646(22,42)$ & $1.667(45)$ & $1.684(62)$ \\
\hline$G^{\mathrm{maj}}(111)$ & $0.409(399)$ & $0.431(21,29)$ & $0.433(22)$ & $0.434(24)$ \\
\hline$G^{\min }(111)$ & $0.378(379)$ & $0.378(80,79)$ & $0.371(73)$ & $\underline{0.364(67)}$ \\
\hline
\end{tabular}

tematically improve upon the LDA we identify it and the lack of knowledge of the atomic structure as limiting factors in studying transport from first principles. Though the atomic structures could be determined theoretically by total energy minimization, the LDA again presents a barrier since it systematically underestimates lattice constants of transition metals in particular of the $3 d$ series. Gradient corrections sometimes yield improvements but unfortunately not systematically so. We conclude that our knowledge of and ability to calculate from first principles Fermi surfaces for bulk magnetic materials such as Fe or Co does not at present justify using a more accurate but substantially more expensive computational scheme than the present one.

\section{B. Ordered interfaces}

$\mathrm{Cu}$ and $\mathrm{Co}$ have slightly different atomic volumes. The equilibrium lattice constant of $\mathrm{Cu}$ is $3.614 \AA$ and of $\mathrm{Co}$ is $3.549 \AA$, assuming an fcc structure. Even in the absence of interface disorder, the lattice spacing will not be homogeneous and will depend on the lattice constant of the substrate on which the sample was grown, on the global and local concentrations of $\mathrm{Cu}$ and $\mathrm{Co}$, and on other details of how the structure was prepared. In principle we could calculate all of this by energy minimization. However, we judge that the additional effort needed is not justified by current experimental knowledge. Instead, we content ourselves with estimating the uncertainty which results from plausible variations in the (interface) structure by considering two limiting cases and one intermediate case. In each case an fcc structure is as- sumed, with lattice constants corresponding to (i) the atomic volume of $\mathrm{Cu}$, (ii) the atomic volume of $\mathrm{Co}$, and (iii) an intermediate case with arithmetic mean of $\mathrm{Cu}$ and $\mathrm{Co}$ atomic volumes.

Our starting point is a self-consistent TB-LMTO SGF calculation $^{44}$ for the interface embedded between semiinfinite $\mathrm{Cu}$ and $\mathrm{Co}$ leads whose potentials and spin-densities were determined self-consistently in separate "bulk" calculations. The charge and spin-densities are allowed to vary in $n_{\mathrm{Cu}}$ layers of $\mathrm{Cu}$ and $n_{\mathrm{Co}}$ layers of Co bounding the interface. The results of these calculations for $\mathrm{Cu} / \mathrm{Co}(111)$ interfaces and the three different lattice constants detailed above are given in Table II for $n_{\mathrm{Cu}}=4, n_{\mathrm{Co}}=4$. In the Cu layers, only tiny moments are induced. Only four layers away from the interface on the Co side, the magnetic moments are seen to be very close to the bulk values. At the interface, where the $d$-bandwidth is reduced as a result of the lower coordination number, the moments are suppressed rather than enhanced. This occurs because the majority-spin $d$ bands are full and their number cannot increase. The width of the free-electron like $s p$ band is less sensitive to the change in coordination and its exchange splitting also changes less. As a result, there is little change in the $s p$ moment. When the $d$-bandwidth is reduced, there is conversion of minority- and majority-spin $s p$ electrons, without loss of the $s p$ moment, to the minorityspin $d$ band with loss of $d$ moment. This picture is supported by the full calculations.

Earlier we saw that an $\sim 2 \%$ change in lattice constant changed the bulk magnetic moment of fcc Co by $2.3 \%$. The effect of changing the basis, from $s p d$ to $s p d f$, was similar. From Table II, the interface moments are seen to behave in a comparable fashion. The magnetic moment of the interface Co atoms decreases by $3.7 \%$, from $1.636 \mu_{B}$ /atom for $a$ $=3.614 \AA$ to $1.578 \mu_{B}$ /atom for $a=3.549 \AA$ for an $s p d$ basis and decreases from $1.578 \mu_{B}$ /atom to $1.526 \mu_{B}$ /atom for an $s p d f$ basis for $a=3.549 \AA$, a change of $3.4 \%$. Thus the $s p$ to $d_{\min }$ conversion is enhanced at the interface by the reduced $d$-bandwidth.

Once the interface potential has been obtained, the transmission matrix can be calculated and the BZ summation carried out. The convergence of this summation, shown in Fig. 4 for a lattice constant of $a=3.614 \AA$ and an spd basis, closely parallels that seen in Fig. 3 and therefore the $k$-summation does not represent a limitation in practice. Converged conductances

$$
G^{\sigma}(\hat{n})=\frac{e^{2}}{h} \sum_{\mu, \nu, \mathbf{k}_{\|}} T_{\mu \nu}^{\sigma}\left(\mathbf{k}_{\|}\right)=\frac{e^{2}}{h} \sum_{\mu, \nu, \mathbf{k}_{\|}}\left|t_{\mu \nu}^{\sigma}\left(\mathbf{k}_{\|}\right)\right|^{2}
$$

are given in the last two rows of Table II. Though we will not concern ourselves in this paper with the application of the formalism we have been developing to a detailed interpretation of experimental observations, it should be noted that even a modest spin-dependence of "bare" interface conductances $(\sim 20 \%)$ can lead to spin-dependent interface resistances differing by a factor of $\sim 3-5$ once account is taken of the finiteness of the conductance of the perfect leads using a formula derived by Schep et al. ${ }^{4}$ 


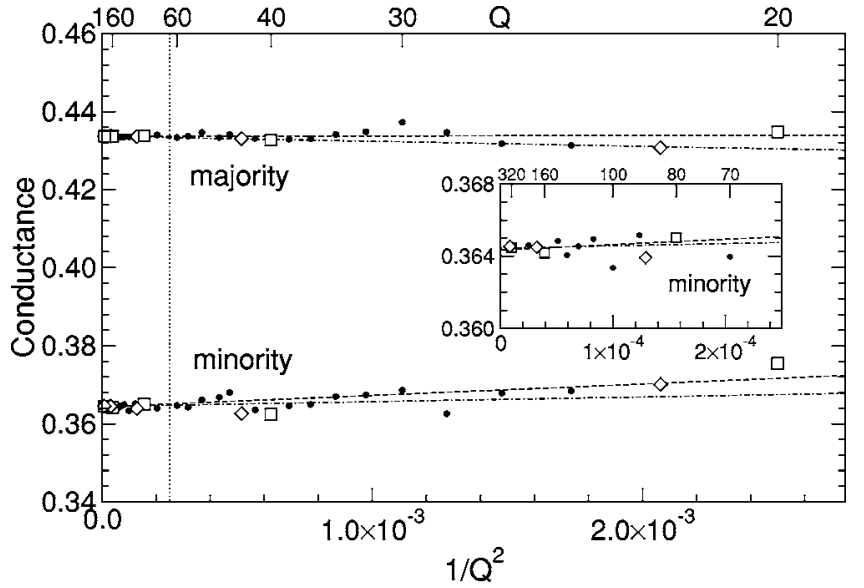

FIG. 4. Interface conductance $G^{\sigma}(111)$ (in units of $10^{15} \Omega^{-1} \mathrm{~m}^{-2}$ ) for an fcc $\mathrm{Cu} / \mathrm{Co}(111)$ interface for majority and minority spins plotted as a function of the normalized area element used in the Brillouin zone summation, $\Delta^{2} \mathbf{k}_{\|} / A_{B Z}=1 / Q^{2}$. $Q$, the number of intervals along the reciprocal lattice vector is indicated at the top of the figure. The dashed line is the weighted (weighting $Q^{2}$ ) least-squares fit to the series $Q=20,40,80,160,320$ shown as squares; the dash-dotted line is the weighted least-squares fit to the series $Q=22,44,88,176,352$ shown as diamonds. The part of the curve for the Co minority spin case to the left of the vertical dotted line is shown on an expanded scale in the inset. A "standard" configuration was used (Ref. 63).

$$
R_{A / B}^{\sigma}=\frac{h}{e^{2}}\left[\frac{1}{\Sigma T_{\mu \nu}^{\sigma}}-\frac{1}{2}\left(\frac{1}{N_{A}^{\sigma}}+\frac{1}{N_{B}^{\sigma}}\right)\right],
$$

where $N_{A}^{\sigma}$ and $N_{B}^{\sigma}$, defined in Eq. (47), are the Sharvin conductances, in units of $e^{2} / h$, of the materials A and B forming the interface.

The majority-spin case can be readily understood in terms of the geometry of the Fermi surfaces of $\mathrm{Cu}$ and $\mathrm{Co}$ so we begin by discussing this simple case before examining the more complex minority-spin channel.

\section{Clean $\mathrm{Cu} / \mathrm{Cu}(111)$ interface: Majority spins}

In the absence of disorder, crystal momentum parallel to the interface is conserved. If, for a given value of $\mathbf{k}_{\|}$, there is a propagating state in $\mathrm{Cu}$ incident on the interface but none in Co, then an electron in such a state is completely reflected at the interface. Conversely, $\mathbf{k}_{\|}$'s for which there is a propagating state in $\mathrm{Co}$ but none in $\mathrm{Cu}$ also cannot contribute to the conductance. To determine the existence of such states, it is sufficient to inspect projections of the Fermi surfaces of fcc $\mathrm{Cu}$ and majority-spin Co onto a plane perpendicular to the transport direction $\hat{n}$, shown in Fig. 5 for $\hat{n}=(111)$. The first feature to note in the figure (left-hand and middle panels) is that per $\mathbf{k}_{\|}$there is only a single channel with positive group velocity so that the transmission matrix in Eq. (48) is a complex number whose modulus squared is a transmission probability with values between 0 and 1 . It is plotted in the right-hand panel and can be interpreted simply. Regions which are depicted blue correspond to $\mathbf{k}_{\|}$'s for which there are propagating states in $\mathrm{Cu}$ but none in $\mathrm{Co}$. These states have transmission probability 0 and are totally reflected. For
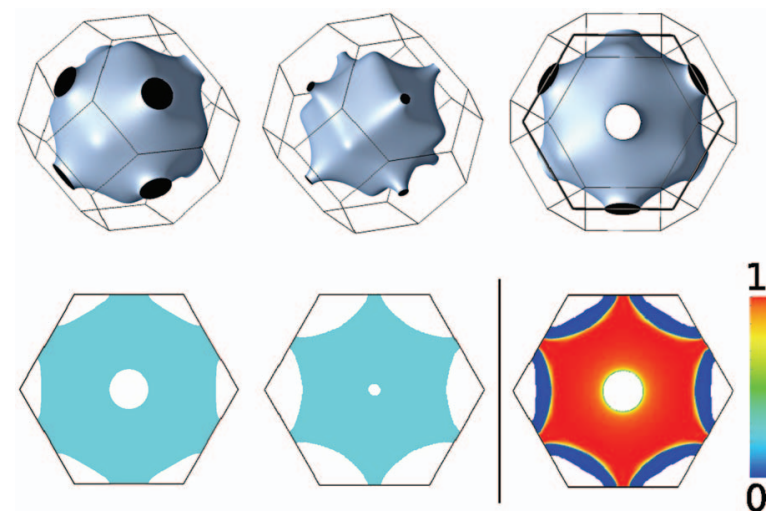

FIG. 5. (Color) Top row, left-hand panel: Fermi surface (FS) of $\mathrm{Cu}$; middle panel: majority-spin FS of Co; right-hand panel: $\mathrm{Cu}$ FS viewed along the (111) direction with a projection of the bulk fcc Brillouin zone (BZ) onto a plane perpendicular to this direction and of the two-dimensional BZ. Bottom row, left-hand and middle panels: projections onto a plane perpendicular to the (111) direction of the $\mathrm{Cu}$ and majority-spin Co Fermi surfaces; right-hand panel: transmission probability for majority-spin states as a function of transverse crystal momentum, $T\left(\mathbf{k}_{\|}\right)$for an fcc $\mathrm{Cu} / \mathrm{Co}(111)$ interface.

values of $\mathbf{k}_{\|}$for which there are propagating states in both $\mathrm{Cu}$ and $\mathrm{Co}$, the transmission probability is very close to one, depicted red. These states are essentially free electronlike states which have the same symmetry in both materials and see the interface effectively as a very low potential step. Close to the center of the figure there is an annular region where there are propagating states in $\mathrm{Co}$ but none in $\mathrm{Cu}$ so they do not contribute to the conductance. Performing the sum in Eq. (48), we arrive at an interface conductance of $0.434 \times 10^{15} \Omega^{-1} \mathrm{~m}^{-2}$ to be compared to the Sharvin conductances given in Table I for $\mathrm{Cu}$ and $\mathrm{Co}$; for $a=3.614 \AA$ and an spd basis these are, respectively, 0.558 and 0.466 in the same units. The interface conductance of 0.434 is seen to be essentially the Sharvin conductance of the majority states of Co reduced because the states closest to the $\Lambda$ axis (corresponding to the symmetry axis of the figures, the $\Gamma L$ line in reciprocal space) do not contribute. The explanation of the $5 \%$ decrease found on going from an spd to an spdf basis (0.431 to 0.409$)$, parallels that given for the corresponding change in the Sharvin conductance of bulk Co (0.469 to 0.449 in Table I).

\section{Clean $\mathrm{Cu} / \mathrm{Cu}(111)$ interface: Minority spins}

The minority-spin case is considerably more complex because the Co minority-spin $d$ bands are only partly filled, resulting in multiple sheets of Fermi surface. These sheets are shown in Fig. 6 together with their projections onto a plane perpendicular to the (111) transport direction. Compared to Fig. 5, one difference we immediately notice is that even single Fermi surface (FS) sheets are not single valued: for a given $\mathbf{k}_{\|}$there can be more than one mode with positive group velocity. The areas depicted green in the projections of the FS sheets from the fourth and fifth bands are examples where this occurs. 

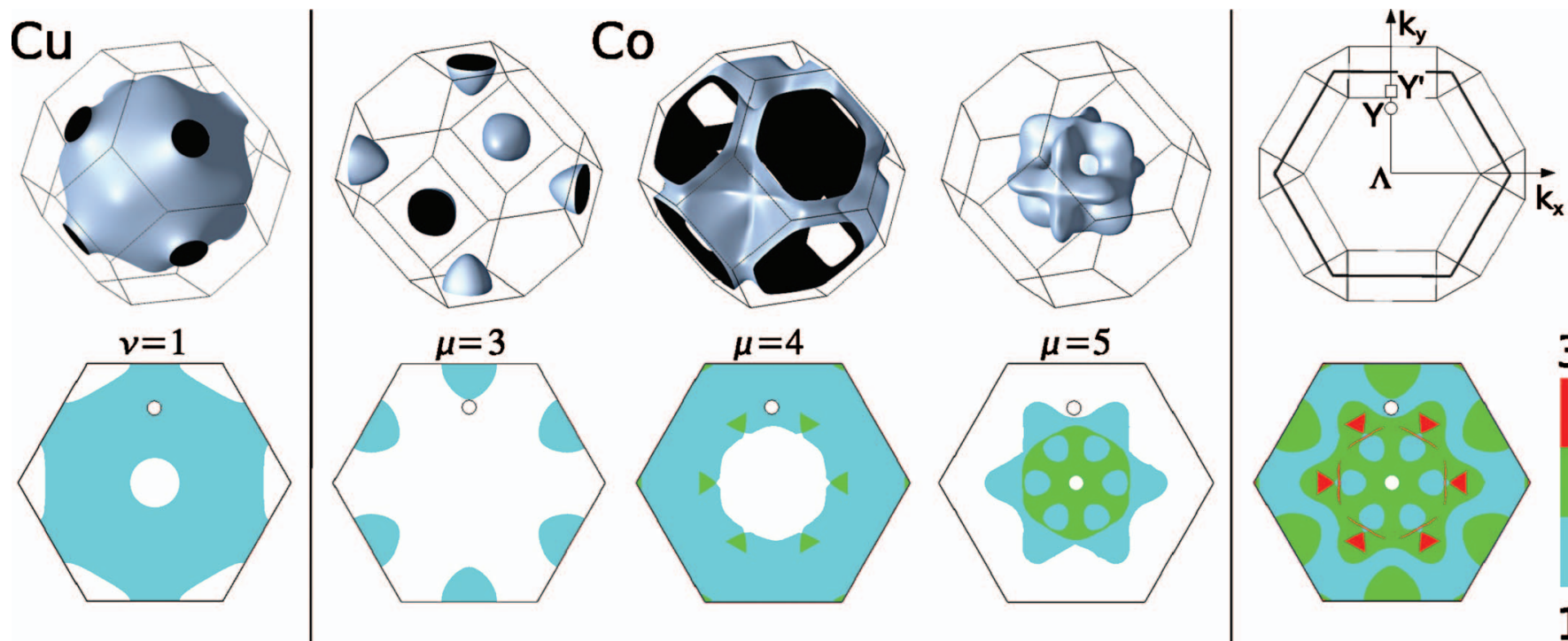

3
1
0
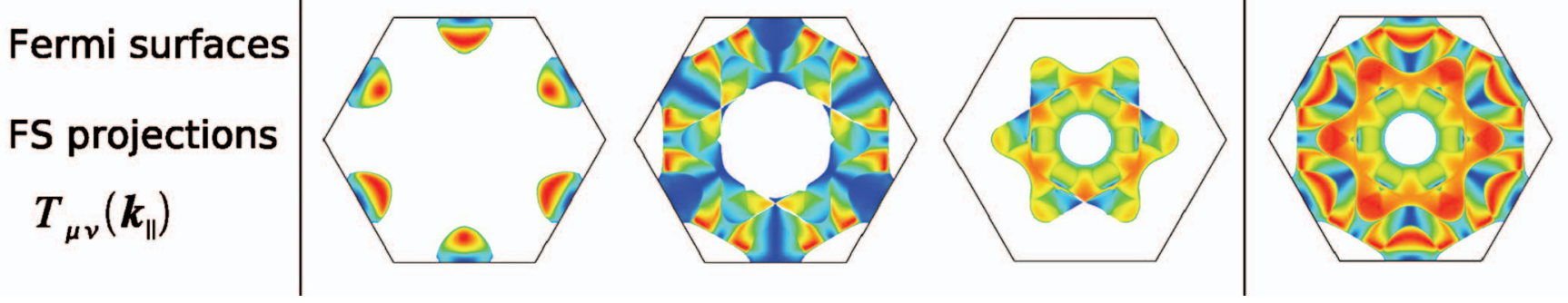

FIG. 6. (Color) Top row, left-hand panel: Fermi surface (FS) of fcc $\mathrm{Cu}$; middle panels: third, fourth, and fifth FS sheets of minority-spin
fcc Co; right-hand panel: projection of the bulk fcc Brillouin zone (BZ) onto a plane perpendicular to the (111) direction and of the two-dimensional BZ. Middle row: corresponding projections of individual FS sheets and (rhs) of Co total. The number of propagating states with positive velocity is color-coded following the color bar on the right. Bottom row: probability $T_{\mu \nu}\left(\mathbf{k}_{\|}\right)$for a minority-spin state on the single FS sheet of $\mathrm{Cu}(\nu=1)$ to be transmitted through a $\mathrm{Cu} / \mathrm{Co}(111)$ interface into FS sheet $\mu$ of fcc Co as a function of the transverse crystal momentum $\mathbf{k}_{\|}$. The point $Y$ (indicated by a small open circle) is such that there are only propagating states in Cu and in the fourth FS sheet of Co. For the point $Y^{\prime}$ (indicated by a small open square) which is slightly further away from $\Lambda$, there is, in addition, a propagating state in the third FS sheet of Co.

An electron incident on the interface from the $\mathrm{Cu}$ side, with transverse crystal momentum $\mathbf{k}_{\|}$, is transmitted into a linear combination of all propagating states with the same $\mathbf{k}_{\|}$ in $\mathrm{Co}$; the transmission matrix $t_{\mu \nu}^{\sigma}\left(\mathbf{k}_{\|}\right)$is in general not square but rectangular. The transmission probabilities $T_{\mu \nu}\left(\mathbf{k}_{\|}\right)$are shown in the bottom row of Fig. 6. Because there is only a single incident state for all $\mathbf{k}_{\|}$, the maximum transmission probability is one. Comparison of the total minority-spin transmission probability $T_{\mathcal{L R}}\left(\mathbf{k}_{\|}\right)$(Fig. 6, bottom right-hand panel) with the corresponding majority-spin quantity (bottom right-hand panel of Fig. 5) strikingly illustrates the spindependence of the interface scattering, much more so than the integrated quantities might have led us to expect; the interface conductances, 0.364 and $0.434 \times 10^{15} \Omega^{-1} \mathrm{~m}^{-2}$ from Table II, differ by only $\sim 20 \%$.

Three factors contribute to the large $\mathbf{k}_{\|}$-dependence of the transmission probability: first and foremost, the complexity of the Fermi surface of both materials but especially of the minority spin of Co; secondly and inextricably linked with the first because of the relationship $\hbar v_{\mathbf{k}}=\nabla_{k} \varepsilon(\mathbf{k})$, the mismatch of the Fermi velocities of the states on either side of the interface. Third, the orbital character of the states $\mu$ and $\nu$ which varies strongly over the Fermi surface and gives rise to large matrix element effects.
The great complexity of transition metal Fermi surfaces, clear from the figure and well-documented in standard textbooks, is not amenable to simple analytical treatment and has more often than not been neglected in theoretical transport studies. Nevertheless, as illustrated particularly well by the ballistic limit, ${ }^{1,37}$ spin-dependent band structure effects have been shown to lead to magnetoresistance ratios comparable to what are observed experimentally in the currentperpendicular-to-plane (CPP) measuring configuration and cannot be simply ignored in any quantitative discussion. Most attempts to take into account contributions of the $d$ states to electronic transport do so by mapping the five $d$ bands onto a single tight-binding or free-electron band with a large effective mass.

Fermi surface topology alone cannot explain all aspects of the tranmission coefficients seen in Fig. 6. For example, there are values of $\mathbf{k}_{\|}$, such as that labeled $Y$ in the figure, for which propagating solutions exist on both sides of the interface yet the transmission probability is zero. This can be understood as follows. At $\mathbf{k}_{\|}=Y$, the propagating states in $\mathrm{Cu}$ have $\left\{s, p_{y}, p_{z}, d_{y z}, d_{3 z^{2}-r^{2}}, d_{x^{2}-y^{2}}\right\}$ character (assuming the choice of in-plane axes as illustrated in the top right-hand panel of Fig. 6) and are even with respect to reflection in the plane formed by the $y$ axis and the transport direction per- 
pendicular to the (111) plane which we choose to be the $z$ axis. For this $\mathbf{k}_{\|}$the only propagating state in Co is in the fourth band. It has $\left\{p_{x}, d_{x y}, d_{x z}\right\}$ character which is odd with respect to reflection in the $y z$ plane. Consequently, the corresponding hopping matrix elements in the Hamiltonian (and in the Green's function) vanish and the transmission is zero.

Along the $k_{y}$ axis the symmetry of the states in $\mathrm{Cu}$ and those in the fourth band of Co remain the same and the transmission is seen to vanish for all values of $k_{y}$. However, at points further away from $\Lambda$, we encounter states in the third band of Co which have even character whose matrix elements do not vanish by symmetry and we see substantial transmission probabilities. Similarly, for points closer to $\Lambda$, there are states in the fifth band of Co with even character whose matrix elements also do not vanish and again the transmission probability is substantial. Because it is obtained by superposition of transmission probabilities from $\mathrm{Cu}$ into the third, fourth, and fifth sheets of the Co FS, the end result, though it may appear very complicated, can be straightforwardly analyzed in this manner $k$-point by $k$-point.

Though the underlying lattice symmetry is only threefold, the Fermi surface projections shown in Fig. 6 have sixfold rotational symmetry about the line $\Lambda$ because the bulk fcc structure has inversion symmetry (and time-reversal symmetry). The interface breaks the inversion symmetry so $T_{\mu \nu}\left(\mathbf{k}_{\|}\right)$ has only threefold rotation symmetry for the individual FS sheets. However, in-plane inversion symmetry is recovered for the total transmission probability $T_{\mathcal{L R}}\left(-\mathbf{k}_{\|}\right)=T_{\mathcal{L} R}\left(\mathbf{k}_{\|}\right)$ which has full sixfold symmetry. This follows from the timereversal symmetry and is proven in Appendix B.

\section{Interface disorder}

Instructive though the study of perfect interfaces may be in gaining an understanding of the role electronic structure mismatch may play in determining giant magnetoresistive effects, all measurements are made on devices which contain disorder, mostly in the diffusive regime. Because there is little information available from experiment about the nature of this disorder, it is very important to be able to model it in a flexible manner, introducing a minimum of free parameters. To model interfaces between materials with different lattice constants and disorder, we use the lateral supercells described in Sec. II D. Since this approach is formally only valid if sufficiently large supercells are used, we begin by studying how the interface conductance depends on the lateral supercell size.

To perform fully self-consistent calculations for a number of large lateral supercells and for different configurations of disorder would be prohibitively expensive. Fortunately, the coherent potential approximation (CPA) is a very efficient way of calculating charge and spin densities for a substitutional disordered $A_{x} B_{1-x}$ alloy with an expense comparable to that required for an ordered system with a minimal unit cell. ${ }^{64}$ The output from such a calculation are atomic sphere potentials for the two sites, $v_{A}$ and $v_{B}$. The layer CPA approximation generalizes this to allow the concentration to vary from one layer to the next. ${ }^{44}$

Once $v_{A}$ and $v_{B}$ have been calculated for some concentration $x$, an $H=H_{1} \times H_{2}$ lateral supercell is constructed in

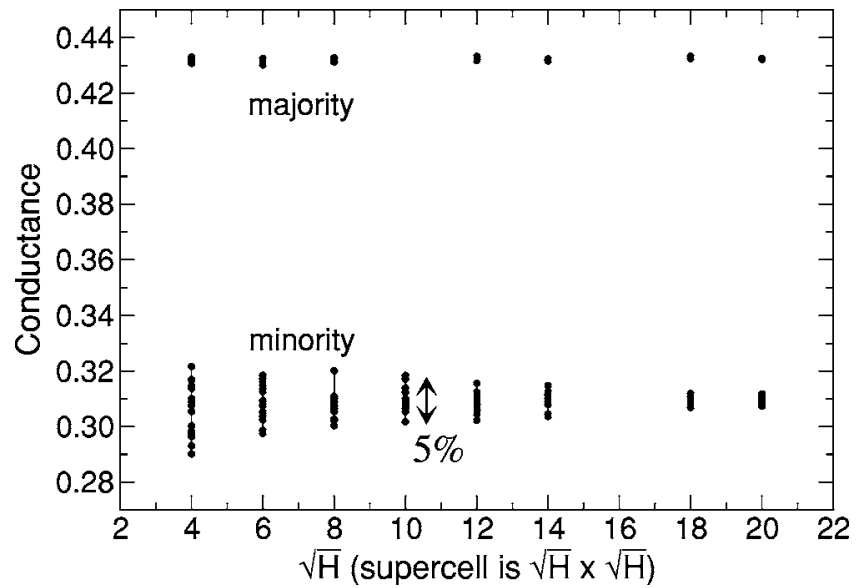

FIG. 7. Interface conductance (in units of $10^{15} \Omega^{-1} \mathrm{~m}^{-2}$ ) for a disordered $\mathrm{Cu} / \mathrm{Co}$ (111) interface modelled as $2 \mathrm{ML}$ of $50 \%-50 \%$ alloy in a $\sqrt{H} \times \sqrt{H}$ lateral supercell as a function of $\sqrt{H}$. The results are given for different randomly generated configurations of disorder (15 for minority spin, 5 for majority spin). Results are for a "standard" configuration (Ref. 63).

which the potentials are distributed at random, maintaining the concentration for which they were self-consistently calculated. The conductances calculated for $4 \leqslant \sqrt{H} \leqslant 20$ are shown in Fig. 7 for a $\mathrm{Cu} / \mathrm{Cu}(111)$ interface in which the $\mathrm{Cu}$ and the Co layers forming the interface are totally mixed to give two layers of 50\%-50\% interface alloy. For each value of $H$, the results for a number of different randomly generated disorder configurations are shown (15 for minority, 5 for majority spin). The sample to sample variation is largest for the minority spin case, ranging from $\pm 5 \%$ for a modest $4 \times 4$ unit cell and decreasing to less than $\pm 1 \%$ for a 20 $\times 20$ unit cell. For $\sqrt{H} \sim 10$, the spread in minority spin conductances is $\sim 5 \%$ which is comparable to the typical uncertainty we associated with the LDA error, the uncertainty in lattice constants or the error incurred by using the ASA.

Comparing now the conductances without and with disorder, we see that interface disorder has virtually no effect on the majority spin channel $(0.434$ versus 0.432 $\times 10^{15} \Omega^{-1} \mathrm{~m}^{-2}$ ) which is a consequence of the great similarity of the $\mathrm{Cu}$ and $\mathrm{Co}$ majority spin potentials and electronic structures. However, in the minority-spin channel the effect $\left(0.364\right.$ versus $\left.0.31 \times 10^{15} \Omega^{-1} \mathrm{~m}^{-2}\right)$ is much larger. As noted in the context of Eq. (49), a relatively small change in the interface transmission can lead to a large change in the interface resistance when account is taken of the finite conductance of the leads. We will return to the consequences for the spin-dependent interface resistance after completing the study of the interface transmission on which it is based.

\section{Dependence of interface conductance on alloy concentration}

The transmission probabilities can be classified as being specular or diffuse depending upon whether or not transverse momentum is conserved. ${ }^{13,65}$ In the presence of interface disorder, modeled here in lateral supercells, the conductance per unit area can be expressed as 


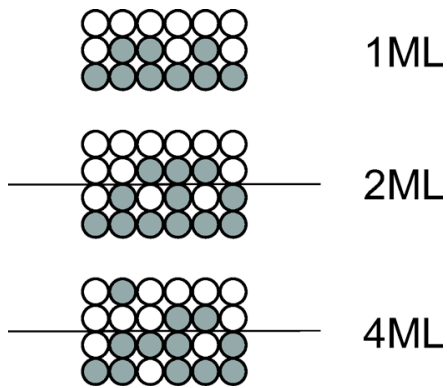

FIG. 8. (Color online) Illustration of three different models of interface disorder considered. Top (1ML): disorder is modeled using one monolayer (1ML) of $\left[\mathrm{Cu}_{1-x} \mathrm{Co}_{x}\right]$ alloy between $\mathrm{Cu}$ and $\mathrm{Co}$ leads, denoted as $\mathrm{Cu}\left[\mathrm{Cu}_{1-x} \mathrm{Co}_{x}\right] \mathrm{Co}$. Middle (2ML): disorder modeled in two MLs as $\mathrm{Cu}\left[\mathrm{Cu}_{1-x} \mathrm{Co}_{x} \mid \mathrm{Cu}_{x} \mathrm{Co}_{1-x}\right]$ Co. Bottom (4ML): starting from the $2 \mathrm{ML}$ disorder case, $1 / 3$ of the concentration $x$ of impurity atoms is transferred to the next layer resulting in $\mathrm{Cu}\left[\mathrm{Cu}_{1-x / 3} \mathrm{Co}_{x / 3} \mid \mathrm{Cu}_{1-2 x / 3} \mathrm{Co}_{2 x / 3}\right.$ $\left.\left|\mathrm{Cu}_{2 x / 3} \mathrm{Co}_{1-2 x / 3}\right| \mathrm{Cu}_{x / 3} \mathrm{Co}_{1-x / 3}\right]$ Co with disorder in four MLs.

$$
G=G_{s}+G_{d}=\frac{e^{2}}{h} \sum_{\substack{\mu \nu \\ \mathbf{k}_{\|}}} T_{\mu \nu}\left(\mathbf{k}_{\|}, \mathbf{k}_{\|}\right)+\frac{e^{2}}{h} \sum_{\substack{\mu \nu \\ \mathbf{k}_{\|} \neq \mathbf{k}_{\|}^{\prime}}} T_{\mu \nu}\left(\mathbf{k}_{\|}, \mathbf{k}_{\|}^{\prime}\right),
$$

where $\mathbf{k}_{\|}$and $\mathbf{k}_{\|}^{\prime}$ belong to the two dimensional Brillouin zone for the $(1 \times 1)$ lateral unit cell and $T_{\mu \nu}\left(\mathbf{k}_{\|}, \mathbf{k}_{\|}^{\prime}\right)$ $=t_{\mu \nu}\left(\mathbf{k}_{\|}, \mathbf{k}_{\|}^{\prime}\right) t_{\mu \nu}^{\dagger}\left(\mathbf{k}_{\|}, \mathbf{k}_{\|}^{\prime}\right)$. The transmission matrix elements between two Bloch states with the same $\mathbf{k}_{\|}$are defined to be specular, those between scattering states with different $\mathbf{k}_{\|}$as being diffuse. In the absence of interface disorder, there is by definition only a specular component.

The results in Fig. 7 were obtained for a structural model of the $\mathrm{Co} / \mathrm{Cu}(111)$ interface consisting of two monolayers (2ML) of $50 \%-50 \%$ alloy that was derived from x-ray, ${ }^{66}$ NMR, ${ }^{67,68}$ and magnetic Extended X-ray Absorption Fine Structure (EXAFS) ${ }^{69}$ studies. Though the most plausible model there is at present, it contains large uncertainties. This makes it important to explore the consequences of varying the parameters defining the model. To do so, we calculate the conductance using $20 \times 20$ lateral supercells as a function of alloy concentration for models in which the disorder is confined to one, two, or four monolayers. The three models are defined in Fig. 8. From the results shown in Fig. 9, it can be seen that the interface transmission for majority-spin electrons depends only very weakly on alloy concentration and its spatial distribution: the results for the $1 \mathrm{ML}, 2 \mathrm{ML}$, and 4ML models cannot be distinguished on the scale of the figure. When the conductance is decomposed using Eq. (50), the diffuse component is found to be very small. Therefore only the results for the minority-spin case need be examined in any detail.

We start by varying the alloy concentration over the full concentration range $(0-100 \%)$ in steps of $10 \%$ for a disordered monolayer. The variation in the total transmission of $\sim 7 \%$ seen in Fig. 9 (1ML), substantially exceeds the spread found for different configurations of disorder (which according to Fig. 7 is less than $\pm 1 \%$ for a $20 \times 20$ lateral supercell) and is therefore statistically significant. Upon adding Co to a

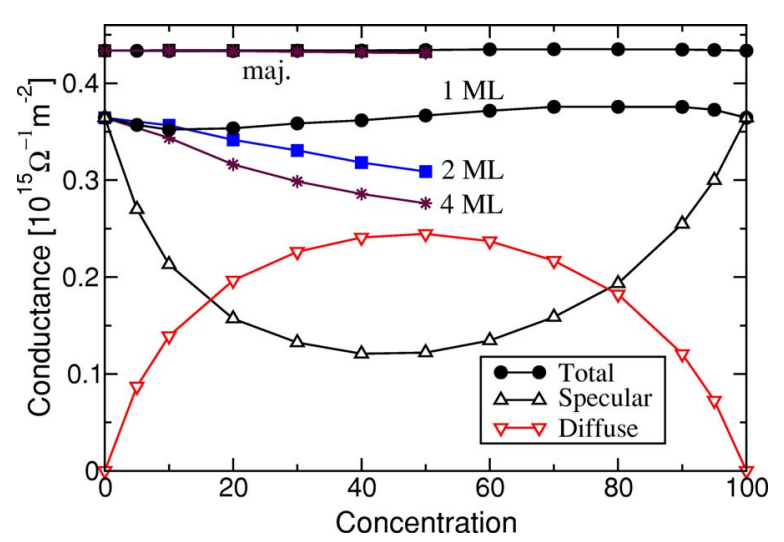

FIG. 9. (Color online) Interface conductance of a disordered $\mathrm{Cu} / \mathrm{Co}(111)$ interface with disorder modeled in a $20 \times 20$ lateral supercell as a function of the concentration $x$ (in percent) of Co in $\mathrm{Cu}$. Results are shown for three different models with disorder in 1 , 2, or 4 MLs which are described in Fig. 8 where $x$ is also defined. Only a single disorder configuration was used and the size of the symbols corresponds to the spread in values found for this supercell size in Fig. 7. For 1ML, the total conductance is resolved into specular and diffuse components. Results are for a "standard" configuration (Ref. 63).

layer of $\mathrm{Cu}$, the transmission decreases, reaches a minimum for $\sim 10 \% \mathrm{Co}$, then increases monotonically up to $\sim 80 \% \mathrm{Co}$ where the transmission is higher than for a clean interface. ${ }^{70}$ $100 \%$ Co represents a clean interface again, so this limit must yield the same transmission as $0 \% \mathrm{Co}$.

The variation can be examined in terms of the specular and diffuse components defined in Eq. (50). From Fig. 9, it can be seen that, for the minority spin channel, the diffuse scattering by $\mathrm{Co}$ impurity atoms in $\mathrm{Cu}$ is stronger than that by $\mathrm{Cu}$ impurity atoms in $\mathrm{Co}$. However, the specular scattering is also more strongly reduced by $\mathrm{Co}$ in $\mathrm{Cu}$ than by $\mathrm{Cu}$ in Co. The two effects largely cancel resulting in the undulatory total transmission as a function of the alloy concentration seen in the figure. The diffuse scattering has a maximum close to a $50 \%-50 \%$ alloy concentration where its contribution to the conductance is almost twice as large as from the specular scattering. While the conductance as such is scarcely affected, the strong diffuse scattering will play an important role in destroying the phase coherence of the electrons, ultimately justifying semiclassical descriptions of transport. ${ }^{71-73}$ Qualitatively similar results for the specular and diffusive components of the transmission have been reported for the (100) orientation in Ref. 24.

If the disorder extends over more than a monolayer, then modeling the interface as several layers of homogeneous alloy is not obviously realistic. Instead, one might expect the layers closest to the interface to be most strongly mixed, the amount of mixing decreasing with the separation from the interface. A simple way to model this is to take two interface layers, one $\mathrm{Cu}$ and one $\mathrm{Co}$, and to mix them in varying degrees. Denoting this $\mathrm{Cu} \mid \mathrm{Co}$ interface as $\mathrm{Cu}\left[\mathrm{Cu}_{1-x} \mathrm{Co}_{x} \mid \mathrm{Cu}_{x} \mathrm{Co}_{1-x}\right] \mathrm{Co}$ we consider $0 \leqslant x \leqslant 0.5$, i.e., the $\mathrm{Cu}$ concentration decreases monotonically from left to right. The calculated interface transmission is seen (Fig. 9, 2ML) to essentially interpolate linearly the results obtained previ- 


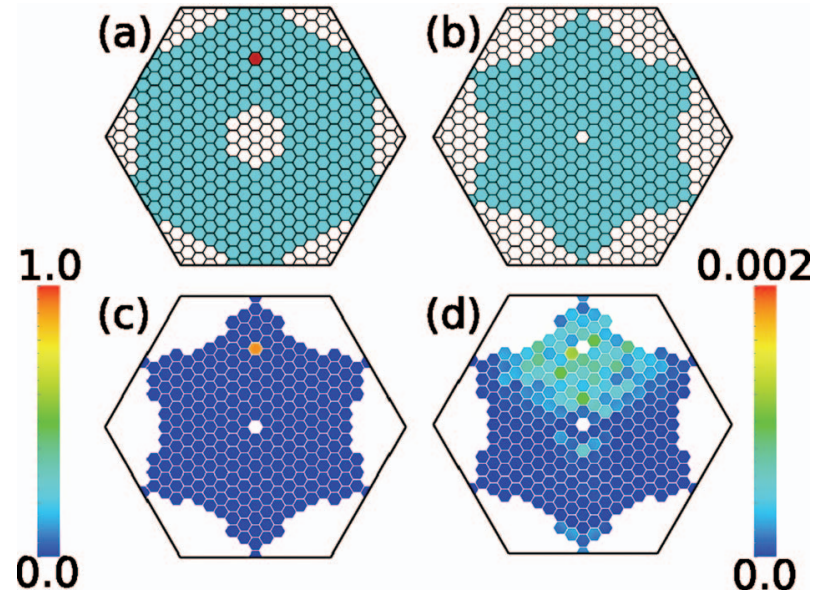

FIG. 10. (Color) Fermi surface projections of majority-spin fcc $\mathrm{Cu}$ (a) and $\mathrm{Co}(\mathrm{b})$ derived from a single $k$-point using a $20 \times 20$ lateral supercell. The dark red point in the $\mathrm{Cu}$ Fermi surface projection corresponds to the point $Y$ in the top right-hand panel of Fig. 6. $T\left(Y, \mathbf{k}_{\|}^{\prime}\right)$ is shown in (c), and in (d) magnified by a factor 500 where the ballistic component $T\left(Y, \mathbf{k}_{\|}^{\prime}=Y\right)$ is indicated by a white point because its value goes off the scale. The results were obtained by averaging over five different configurations of disorder.

ously for the clean $(x=0)$ and disordered $(x=0.5)$ cases.

A slightly more elaborate model can be constructed from the $2 \mathrm{ML}$ model by distributing the $x$ impurity atoms so that $2 x / 3$ are in the interface layer while $x / 3$ are to be found further from the original interface, in the following layer. This results in the concentration profile $\mathrm{Cu}\left[\mathrm{Cu}_{1-x / 3} \mathrm{Co}_{x / 3}\left|\mathrm{Cu}_{1-2 x / 3} \mathrm{Co}_{2 x / 3}\right| \mathrm{Cu}_{2 x / 3} \mathrm{Co}_{1-2 x / 3}\right.$ $\left.\mid \mathrm{Cu}_{x / 3} \mathrm{Co}_{1-x / 3}\right]$ Co. $x=0$ corresponds to a completely ordered interface while the maximum value $x$ can have so that the concentration decreases from left to right monotonically is $75 \%$. This relatively small redistribution of intermixed atoms is seen to reduce the transmission by $15 \%$ for $x=0.5$ (Fig. 9, $4 \mathrm{ML})$. A detailed analysis of the different contributions to the interface scattering in the $2 \mathrm{ML}$ and $4 \mathrm{ML}$ cases will be given in a separate publication.

\section{Analysis of interface disorder scattering}

The scattering induced by two layers of $50 \%-50 \%$ alloy is illustrated in Figs. 10 and 11 for the majority and minority spins, respectively, of a $\mathrm{Cu} / \mathrm{Co}(111)$ interface. Calculations were performed for the single $\mathbf{k}_{\|}^{\mathrm{S}}$ point, $\Gamma$, and a $20 \times 20$ lateral supercell equivalent to using a $1 \times 1$ interface cell and $k$-space sampling with $20 \times 20$ points in the corresponding BZ. Disorder averaging was carried out using 5 (for majority spin) or 20 (for minority spin) disorder configurations generated randomly.

Figures 10(a) and 10(b) show the majority-spin Fermi surface projections of fcc $\mathrm{Cu}$ and $\mathrm{Co}$, respectively, obtained from "unfolding" the supercell calculation. The coarse 20 $\times 20$ grid is seen to yield a good representation of the detailed Fermi surface projections shown in Fig. 5. $T\left(\mathbf{k}_{\|}, \mathbf{k}_{\|}^{\prime}\right)$ is shown in Fig. 10(c) for $\mathbf{k}_{\|}=Y$ on the $k_{y}$ axis in Fig. 6. Specular scattering dominates with $T_{s}(Y)=T\left(\mathbf{k}_{\|}=Y, \mathbf{k}_{\|}^{\prime}=Y\right)=0.93$.
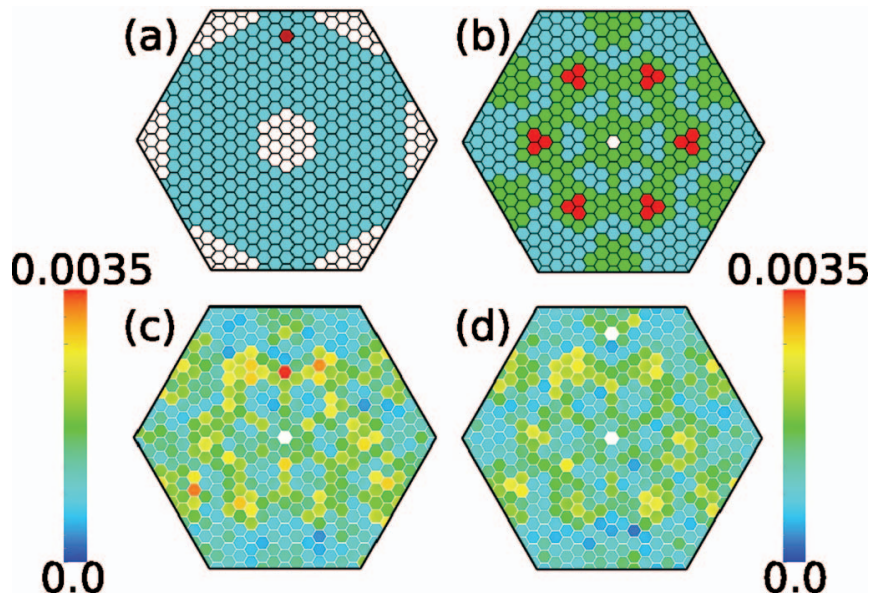

FIG. 11. (Color) Fermi surface projections of minority-spin fcc $\mathrm{Cu}$ (a) and $\mathrm{Co}(\mathrm{b})$ derived from a single $k$-point using a $20 \times 20$ lateral supercell. The dark red point in the $\mathrm{Cu}$ Fermi surface projection corresponds to the point $Y^{\prime}$ in the top right-hand panel of Fig. 6. (c) $T\left(Y, \mathbf{k}_{\|}^{\prime}\right)$ and (d) $T\left(Y^{\prime}, \mathbf{k}_{\|}^{\prime}\right)$ calculated using 20 different disorder configurations; the ballistic component $T\left(Y^{\prime}, \mathbf{k}_{\|}^{\prime}=Y^{\prime}\right)$ is indicated by a white point because its value goes off scale. The results were obtained by averaging over 20 different configurations of disorder.

The diffuse scattering is so weak that nothing can be seen on a scale of $T$ from 0 to 1 . To render it visible, a magnification by a factor 500 is needed, Fig. 10(d). The total diffuse scattering $T_{d}(Y)=\Sigma_{k_{\|}^{\prime} \neq Y} T\left(Y, \mathbf{k}_{\|}^{\prime}\right)=0.04$ can be seen from the figure to be made up of contributions of $T \sim 0.0004$ from roughly a quarter of the BZ (100 $\mathbf{k}_{\|}$points) centered on $\mathbf{k}_{\|}$ $=Y$. The total transmission, $T_{\text {total }}=T_{s}+T_{d}=0.93+0.04=0.97$, compared to a transmission of 0.99 in the absence of disorder. Similar results were obtained for other $\mathbf{k}_{\|}$points. In the majority case, there is thus a strong specular peak surrounded by a weak diffuse background.

The minority-spin Fermi surface projections of fcc $\mathrm{Cu}$ and Co are shown in Figs. 11(a) and 11(b), respectively. Compared to the corresponding panels in Fig. 6, the $20 \times 20$ point representation is seen to be sufficient to resolve the individual Fermi surface sheets of Co. To study the effect of interface disorder, we consider scattering out of two different $\mathbf{k}_{\|}$'s in $\mathrm{Cu}$ [Figs. 11(c) and 11(d)]. The first thing to note is the similarity of both transmission plots to the projected FS of Co, Fig. 11(b), suggesting very strong diffusive scattering proportional to the density of available final states.

The first case we consider is where $\mathbf{k}_{\|}=Y$ for which the transmission was zero as a result of the symmetry of the states along the $k_{y}$ axis in the absence of disorder. $T\left(Y, \mathbf{k}_{\|}^{\prime}\right)$ is shown in Fig. 10(c). By contrast with the majority-spin case just examined, there is now scattering to all other $k$-points in the 2D BZ, $\sum_{k_{\|}^{\prime} \neq Y} T\left(Y, \mathbf{k}_{\|}^{\prime}\right)=0.58$ while $T(Y, Y)$ has only increased from 0.00 in the clean case, to 0.01 in the presence of disorder. The effect of disorder is to increase the total transmission, $T_{\text {total }}(Y)=\Sigma_{k_{\|}^{\prime}} T\left(Y, \mathbf{k}_{\|}^{\prime}\right)$ from 0.00 to $T_{s}(Y)$ $+T_{d}(Y)=0.01+0.58=0.59$; for states which were originally strongly reflected, disorder increases the transmission.

The second case we consider is that of a $k$-point slightly further away from the origin $\Lambda$ along the $k_{y}$ axis which had a 


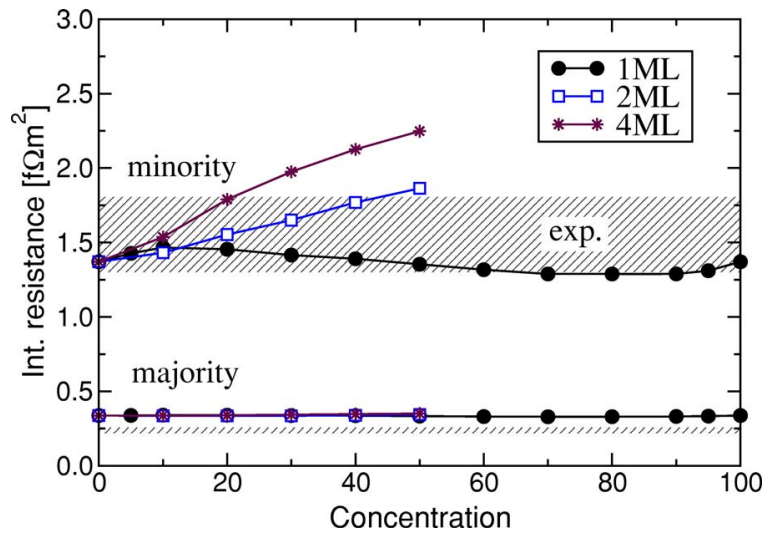

FIG. 12. (Color online) Interface resistance for disordered interfaces as a function of the alloy concentration used to model disordered interfaces calculated using Eq. (49) and the transmission probabilities shown in Fig. 9. The experimental values for sputtered and MBE grown multilayers cited in Table I of Ref. 76 span a range of values which is indicated by the shaded regions.

high transmission, $T\left(Y^{\prime}\right)=0.98$, in the absence of disorder. For this $k$-point, $T\left(Y^{\prime}, \mathbf{k}_{\|}^{\prime}\right)$, shown in Fig. 10(d), looks very similar to Fig. 10(c). There is strong diffuse scattering with $\Sigma_{k_{\|}^{\prime} \neq Y^{\prime}} T\left(Y^{\prime}, \mathbf{k}_{\|}^{\prime}\right)=0.54$ while $T\left(Y^{\prime}, Y^{\prime}\right)$ has been drastically decreased from 0.98 in the clean case, to 0.06 as a result of disorder. The total transmission, $T_{\text {total }}\left(Y^{\prime}\right)=T_{s}\left(Y^{\prime}\right)+T_{d}\left(Y^{\prime}\right)$ $=0.06+0.54=0.60$, is almost identical to what was found for the $Y$ point. The effect of disorder has been to decrease the transmission for states which were originally weakly reflected. The strong $k$-dependence of the transmission found in the specular case is largely destroyed by a small amount of disorder in the minority-spin channel. The contribution from the specular component (integrated over 2D BZ) is reduced to $15 \%$ of the total transmission.

\section{E. Interface resistance}

To the best of our knowledge, spin-dependent interface transmissions have not yet been measured directly. What is usually done $\mathrm{e}^{74,75}$ is to measure total resistances for a whole series of magnetic multilayers in which the total number of interfaces and/or the thicknesses of the individual layers is varied. The measured results are interpreted in terms of volume resistivities and interface resistances. By applying an external magnetic field, the magnetizations of neighboring layers which are oriented antiparallel (AP) can be forced to line up in parallel (P). By measuring the resistances in both cases, spin-dependent volume resistivities and interface resistances can be extracted using the two current series resistor (2CSR) model. ${ }^{71-73}$ If we take expression (49) which relates the interface transmission to the interface resistance occurring in the 2CSR model as given, ${ }^{4,34}$ we can study how typical uncertainties in interface transmission, arising from arbitrary assumptions about the interface disorder, lattice constant, or basis set translate into uncertainty in predicted interface resistances. Using the transmission probabilities from Fig. 9 in Eq. (49) results in the curves shown in Fig. 12. For comparison, a range of literature values for the spin-
TABLE III. Interface resistances, in units of $\mathrm{f} \Omega \mathrm{m}^{2}$, for ordered interfaces, calculated using expression (49) and the data from Tables I and II. The values given here for a lattice constant of $a$ $=3.549 \AA$ and $s p d$ basis differ slightly from those reported in Ref. 9 which were performed using energy-independent muffin-tin orbitals linearized about the centers of gravity of the occupied conduction states and not at the Fermi energy. The current implementation (Ref. 49) uses energy-dependent, (nonlinearized) MTOs, calculated exactly at the Fermi energy which improves the accuracy at no additional cost.

\begin{tabular}{|c|c|c|c|}
\hline \multirow{2}{*}{$\begin{array}{l}a(\AA) \\
\text { Basis }\end{array}$} & \multicolumn{2}{|c|}{3.549} & \multirow{2}{*}{$\begin{array}{c}3.614 \\
\text { spd }\end{array}$} \\
\hline & spdf & spd & \\
\hline$R^{\mathrm{maj}}(111)$ & 0.46 & 0.39 & 0.34 \\
\hline$R^{\min }(111)$ & 1.33 & 1.32 & 1.37 \\
\hline
\end{tabular}

dependent interface resistances derived from experiments on sputtered and molecular beam exitaxy (MBE) grown multilayers ${ }^{76}$ is included in the figure.

For the minority-spin case, experimental values (in units of $\mathrm{f} \Omega \mathrm{m}^{2}$ ) range from 1.30 to 1.80 compared to calculated values of 1.29 for $\mathrm{Cu}\left[\mathrm{Cu}_{0.3} \mathrm{Co}_{0.7}\right] \mathrm{Co}$, through 1.37 for a disorder-free interface, to a value of 2.25 for the $4 \mathrm{ML}$ model with $x=0.5, \mathrm{Cu}\left[\mathrm{Cu}_{0.83} \mathrm{Co}_{0.17} \mid \mathrm{Cu}_{0.67} \mathrm{Co}_{0.33}\right.$ $\left.\left|\mathrm{Cu}_{0.33} \mathrm{Co}_{0.67}\right| \mathrm{Cu}_{0.17} \mathrm{Co}_{0.83}\right] \mathrm{Co}$. The influence of lattice constant and basis set on the clean interface resistance values is small (see Table III). The present modeling of interface alloying shows that the interface resistance is more strongly dependent on the detailed spatial distribution of disorder than was previously found ${ }^{9}$ where only the concentration range $x=0.5 \pm 0.06$ of the $2 \mathrm{ML}$ interface alloy model extracted from experiment ${ }^{66-69}$ was explored.

For the majority-spin case, the spread in values of the interface resistance extracted from experiment (for the same samples as for the minority-spin case) is quite small, 0.220.25 , and does not overlap with the value of 0.34 found for a lattice constant of $a=3.614 \AA$. Unlike the minority-spin case, changing the lattice constant or using an spdf basis leads to substantially larger values (Table III). Because the majorityspin transmission does not depend on the details of the interface disorder, this cannot be the origin of the discrepancy.

Motivated by the weak scattering in this case, we examine the validity ${ }^{73,76-79}$ of the 2 CSR model by calculating the resistance of a magnetic multilayer containing a large number of disordered interfaces and plot the resistance added by each additional interface in Fig. 13. Compared to similar calculations in Ref. 9, the number of interfaces, size of lateral supercell $(10 \times 10)$, and disorder configurations averaged over are increased substantially. While the calculations are in very good agreement with Ohm's law for the strongly scattering minority-spin case, it can be seen that this is not the case for the majority-spin electrons. For a small number of interfaces there is a clear breakdown of Ohm's law and thus of the 2CSR model. The interface resistance eventually saturates at a value much lower than those extracted from experiment. While inclusion of bulk scattering will modify this picture somewhat, exploratory calculations ${ }^{80}$ indicate that the type of 


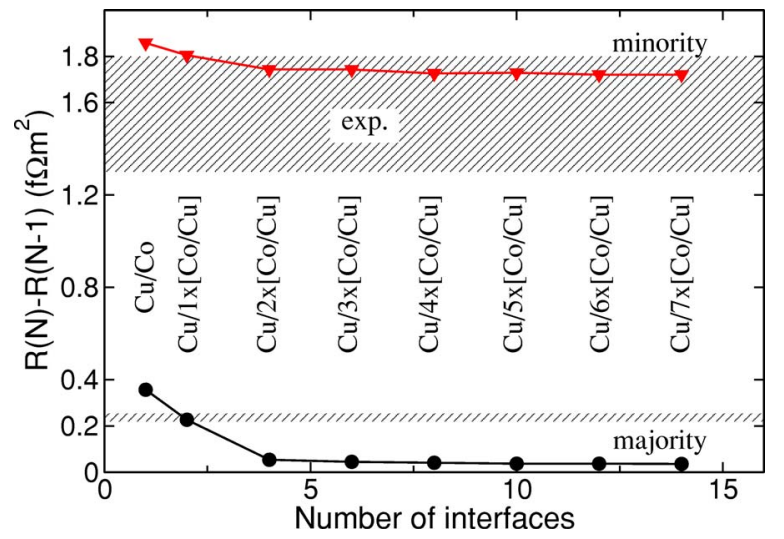

FIG. 13. (Color online) Differential interface resistance as the number of interfaces increase for a disordered $\mathrm{Cu}(10 \mathrm{ML}) / \mathrm{Co}(10 \mathrm{ML})$ multilayer embedded between $\mathrm{Cu}$ leads. A $10 \times 10$ (111) lateral supercell was used and the interface was modeled as two layers of $50 \%-50 \%$ alloy (2ML model). The results represent an average over five disorder configurations and were obtained for a "standard" configuration (Ref. 63). The range of experimental values (Ref. 76) is indicated by the shaded regions.

"bulk" impurities which may be reasonably expected to be found in sputtered or MBE grown multilayers affect the minority spin electrons much more than the majority spins. Agreement for the latter can only be achieved at the expense of ruining good agreement for the former.

\section{DISCUSSION}

Details of a muffin-tin-orbital-based method suitable for calculating from first-principles scattering matrices involving layered magnetic materials have been given. In a wide range of applications, ${ }^{9,46-51}$ it has been shown to be much more efficient and transparent than a previously used LAPW-based method. ${ }^{4,6,38}$ Various other schemes have been developed for calculating the transmission of electrons through an interface (or a more extended scattering region) both from first principles, ${ }^{7,8,10-12,14,15,17,18,35,36,38,81}$ or using as input electronic structures which were calculated from first principles. ${ }^{19,21,22,82-87}$ Most are based upon a formulation for the conductance in terms of nonequilibrium Green's functions ${ }^{58}$ (NEGF) which reduces in the appropriate limit to the well-known Fisher-Lee (FL) linear-response form ${ }^{27}$ for the conductance of a finite disordered wire embedded between crystalline leads. Most implementations of the NEGF or FL schemes have two disadvantages. (i) The transmission is calculated for a complex energy which leads to difficulties in studying, for example, tunneling magnetoresistance, where the finite imaginary part can give rise to an exponential decay which obscures the interesting physical decay of the transmission as a function of the barrier thickness. (ii) For a given value of transverse crystal momentum, the transmission is expressed as a trace over the basis set in terms of which the Green's function and self-energy are expressed. ${ }^{56}$ While this has the advantage that the total transmission can be calculated without explicitly determining the scattering states and can be computationally efficient, summation of the contributions from multiple scattering states can obscure real physical effects, for example, the role of the symmetries of individual scattering states seen in Fig. 6. Explicit determination of the scattering states not only makes a detailed analysis of the scattering possible; the full scattering matrix, expressed in terms of the scattering states, can be used to bridge $^{48}$ the gap between first-principles electronic structure calculations and phenomenological models of transport used to analyze complex situations where a full first-principles treatment is not practical.

We have instead made use of an alternative technique, suitable for Hamiltonians that can be represented in tightbinding form, that was formulated by $\mathrm{Ando}^{45}$ and is based upon direct matching of the scattering-region wave function to the Bloch modes of the leads. The relationship between the wave-function matching ${ }^{45}$ and Green function ${ }^{27,58}$ approaches is not immediately obvious. It was suggested recently that WFM was incomplete ${ }^{88}$ but the equivalence of the two approaches could be proven. ${ }^{56}$ Schemes similar in spirit to our own, but based upon empirical tight-binding Hamiltonians have been presented by Sanvito et al..$^{22}$ and by Velev. ${ }^{23,24}$ In contrast to these schemes, our TB-MTO formalism is a parameter-free approach that has an advantage of self-consistent determination of potentials (on CPA level for disordered systems) and spin densities for systems for which these are not known from experiment. Even though all tightbinding schemes should be fundamentally similar in performance, it would seem, judging from the size of systems to which it has been applied, that our implementation is nevertheless substantially more efficient than these empirical schemes. The scattering regions treated in Figs. 7 and 9-11 contained as many as 3200 atoms $(20 \times 20$ lateral supercell $\times 8$ principal layers where the potential was allowed to deviate from its bulk values) or, in the case of Fig. 13, 15 000 atoms $(10 \times 10$ lateral supercell $\times 150$ principal layers $)$. Our WFM scheme should not be confused ${ }^{24}$ with a recently developed transport formalim ${ }^{8,13}$ also based upon TB-LMTOs but which makes use of the Caroli NEFG expression for the conductance in terms of a trace and a complex energy. Khomyakov and Brocks ${ }^{81}$ have developed a scheme analogous to ours but based upon pseudopotentials and a real space grid which make it more suitable for studying quantum wires or the type of open structures studied in molecular electronics, but is computationally much more expensive.

A third approach based upon "embedding" 89,90 has been combined with the full-potential linearized augmented plane wave method to yield what is probably the most accurate scheme to date ${ }^{14,15,38}$ but like the real space grid WFM method, ${ }^{81}$ these methods are numerically very demanding.

\section{SUMMARY}

Details of a wave-function matching method suitable for calculating the scattering matrices in magnetic metallic hybrid structures based upon first-principles tight-binding muffin-tin orbitals have been given and illustrated with calculations for a variety of $\mathrm{Co} / \mathrm{Cu}(111)$ interface-related problems. The minimal basis of localized orbitals is very efficient, allowing large lateral supercells to be handled. This 
allows us to model materials with large lattice mismatch or to study transport in the diffusive regime. Because the scattering states are calculated explicitly, the effect of various types of scattering can be analyzed in detail.

\section{ACKNOWLEDGMENTS}

This work is part of the research program for the "Stichting voor Fundamenteel Onderzoek der Materie" (FOM) and the use of supercomputer facilities was sponsored by the "Stichting Nationale Computer Faciliteiten" (NCF), both financially supported by the "Nederlandse Organisatie voor Wetenschappelijk Onderzoek" (NWO). It was also supported by the European Commission's Research Training Network Computational Magnetoelectronics (Contract No. HPRNCT-2000-00143) as well as by the NEDO International Joint Research program Nano-scale Magnetoelectronics. K.X. wishes to thank NSF of China for Grant No. 90303014. M.Z. wishes also to acknowledge support from KBN Grant No. PBZ-KBN-044/P03-2001. We are grateful to Ilya Turek for his TB-LMTO-SGF layer code which we used to generate self-consistent potentials and for numerous discussions about the method and Anton Starikov for permission to use his version of the TB-MTO code based upon sparse matrix techniques with which we performed some of the calculations.

\section{APPENDIX A: VELOCITIES}

Let us start by deriving the expression for the group velocity of an eigenstate of a general tight-binding Hamiltonian, which for a translationally invariant system can be written in the Bloch representation as

$$
\mathcal{H}_{R L, R^{\prime} L^{\prime}}(\mathbf{k})=\sum_{\mathbf{T}} e^{i \mathbf{k} \cdot \mathbf{T}} \mathcal{H}_{R L,\left(R^{\prime}+T\right) L^{\prime}},
$$

where $R L(L=l m)$ labels the sites and orbitals within the unit cell and $\mathbf{T}$ runs over lattice vectors. The energy eigenvalues $\varepsilon_{\mu}(\mathbf{k})$ are the expectation values

$$
\varepsilon_{\mu}(\mathbf{k})=\mathbf{a}_{\mu}^{\dagger}(\mathbf{k}) \mathcal{H}(\mathbf{k}) \mathbf{a}_{\mu}(\mathbf{k})
$$

where the eigenvectors $\mathbf{a}_{\mu}(\mathbf{k})$ are indexed by $R L$ and we assume the normalization $\mathbf{a}_{\mu}^{\dagger} \cdot \mathbf{a}_{\mu}=1$. It is now straightforward to calculate the group velocity of the propagating mode

$$
\boldsymbol{v}_{\mu}=\frac{1}{\hbar} \frac{\partial \varepsilon_{\mu}(\mathbf{k})}{\partial \mathbf{k}}=\frac{i}{\hbar} \sum_{\mathbf{T}} \mathbf{T} e^{i \mathbf{k} \cdot \mathbf{T}} \times \sum_{R L, R^{\prime} L^{\prime}} a_{R L}^{*} \mathcal{H}_{R L,\left(R^{\prime}+T\right) L^{\prime}} a_{R^{\prime} L^{\prime}} .
$$

In the mixed representation $\left|I, \mathbf{k}_{\|}\right\rangle$defined in Sec. II B, Eq. (A3) gives for the velocity in the stacking direction

$$
v_{\mu}=\frac{i d}{\hbar}\left[\mathbf{a}_{\mu}^{\dagger} \mathcal{H}_{I, I+1}\left(\mathbf{k}_{\|}\right) \lambda_{\mu} \mathbf{a}_{\mu}-\text { H.c. }\right]
$$

where $d$ is the distance between equivalent monolayers in adjacent principal layers (PL), the hopping is assumed (as in Sec. II B) to extend only between neighboring PLs and $\lambda_{\mu}=\exp \left(i \mathbf{k} \cdot \mathbf{T}^{0}\right)$ with $\mathbf{T}^{0}$ connecting equivalent sites in the neighboring PLs.
Equations (A3) and (A4) are nothing other than the expectation values of a standard tight-binding velocity operator, ${ }^{8,91}$ which can be written in second quantization form as

$$
\hat{v}=\frac{i}{\hbar} \sum_{T, T^{\prime}}\left(\mathbf{T}^{\prime}-\mathbf{T}\right) \times \sum_{R L, R^{\prime} L^{\prime}} \mathcal{H}_{(R+T) L,\left(R^{\prime}+T^{\prime}\right) L^{\prime}} \hat{c}_{(R+T) L}^{\dagger} \hat{c}_{\left(R^{\prime}+T^{\prime}\right) L^{\prime}}
$$

The velocity defined in this way describes the motion of an electron between neighboring unit cells, i.e., the motion related to the overall current carried by the state. Using the relation between the generalized Bloch matrices (24) and the surface Green function one can, equivalently, express Eq. (A3) using the latter quantities. More details can be found in Ref. 56.

In the screened representation, the KKR tail-cancellation equation of Sec. II has the algebraic structure of a tight binding Hamiltonian, but is nonlinear in energy. In order to find equivalents of Eqs. (A3) and (A4) for our MTO formalism, we must relate it to the linearized MTO (LMTO) Hamiltonian. Introducing the Hermitian matrix ${ }^{41,42}$

$$
\begin{aligned}
h^{\alpha}(\varepsilon) & =-\left[\dot{P}^{\alpha}(\varepsilon)\right]^{-1 / 2}\left[P^{\alpha}(\varepsilon)-S^{\alpha}\right]\left[\dot{P}^{\alpha}(\varepsilon)\right]^{-1 / 2} \\
& =-P^{\alpha}(\varepsilon)\left[\dot{P}^{\alpha}(\varepsilon)\right]^{-1}+\left[\dot{P}^{\alpha}(\varepsilon)\right]^{-1 / 2} S^{\alpha}\left[\dot{P}^{\alpha}(\varepsilon)\right]^{-1 / 2},
\end{aligned}
$$

fixing the energy at $\varepsilon=\varepsilon_{F}$, and defining the potential parameters $^{42,43}$

$$
\sqrt{d^{\alpha}}=\left[\dot{P}^{\alpha}\left(\varepsilon_{F}\right)\right]^{-1 / 2}
$$

and

$$
c^{\alpha}=-P^{\alpha}\left(\varepsilon_{F}\right) / \dot{P}^{\alpha}\left(\varepsilon_{F}\right)+\varepsilon_{F},
$$

we arrive at

$$
h^{\alpha} \equiv h^{\alpha}\left(\varepsilon_{F}\right)=c^{\alpha}+\sqrt{d^{\alpha}} S^{\alpha} \sqrt{d^{\alpha}}-\varepsilon_{F} .
$$

Equation (A8) has the form of a two-center tight binding Hamiltonian whose energy is given relative to $\varepsilon_{F}$. It provides the lowest order approximation ${ }^{41,42}$ to the full LMTO Hamiltonian and yields eigenvalues correct to first order in $\left(\varepsilon-\varepsilon_{F}\right)$. Consequently the error introduced by using Eq. (A8) in Eq. (A3) is also of the first order and vanishes for states at the Fermi energy. For such states the eigenvectors of Eq. (A8) are equal to those of Eq. (15) up to the (position and orbital dependent) scaling factor:

$$
\mathbf{c}_{\mu}\left(\mathbf{k}_{\|}\right)=\left[\dot{P}^{\alpha}\left(\varepsilon_{F}\right)\right]^{-1 / 2} \mathbf{a}_{\mu}\left(\mathbf{k}_{\|}\right) .
$$

Using Eqs. (A6) and (A9) in Eq. (A4) we arrive at Eq. (38).

\section{APPENDIX B: SYMMETRY RELATIONS}

If we look closely at the transmission probabilities in Fig. 6 , we see that the sheet resolved transmissions exhibit the geometrical symmetry of the underlying lattice (i.e., the threefold rotational axis). The total transmission probability on the other hand possesses an extra inversion symmetry, 
$T\left(\mathbf{k}_{\|}\right)=T\left(-\mathbf{k}_{\|}\right)$, which results in plots with a sixfold rotational axis. This higher symmetry is the manifestation of the fundamental time-reversal symmetry obeyed in the absence of spin-orbit coupling and a magnetic field. In the case of the bulk system, time-reversal symmetry grants that for every eigenstate $\psi_{\alpha}(\mathbf{k})$ there exists the counterpart with the same energy and opposite wave vector [i.e., $\left.\varepsilon_{\alpha}(\mathbf{k})=\varepsilon_{\alpha}(-\mathbf{k})\right]$ and the wave functions are related by the complex conjugate. The situation is more complicated in the case of the scattering state. Consider a state incoming from the left lead and scattered in the middle region. The wave function consists then of the incoming and reflected states in the left lead

$$
\Psi_{\mathcal{L}}^{r}\left(\mathbf{k}_{\|}\right)=\psi_{\mu}^{+}\left(\mathbf{k}_{\|}\right)+\sum_{\mu^{\prime}} r_{\mu^{\prime} \mu}\left(\mathbf{k}_{\|}\right) \overline{\psi_{\mu^{\prime}}^{-}}\left(\mathbf{k}_{\|}\right)
$$

and of the transmitted states in the right lead

$$
\Psi_{\mathcal{R}}^{r}\left(\mathbf{k}_{\|}\right)=\sum_{\nu} t_{\nu \mu}\left(\mathbf{k}_{\|}\right) \psi_{\nu}^{+}\left(\mathbf{k}_{\|}\right)
$$

The time reversal operation transforms the above "retarded" state into the "advanced" one in which a number of incoming states (from the left and the right) combine to produce a single outgoing state on the left, i.e.,

$$
\Psi_{\mathcal{L}}^{a}\left(-\mathbf{k}_{\|}\right)=\sum_{\mu^{\prime}} r_{\mu^{\prime} \mu}^{*}\left(\mathbf{k}_{\|}\right) \psi_{\mu^{\prime}}^{+}\left(-\mathbf{k}_{\|}\right)+\psi_{\mu}^{-}\left(-\mathbf{k}_{\|}\right)
$$

and

$$
\Psi_{\mathcal{R}}^{a}\left(-\mathbf{k}_{\|}\right)=\sum_{\nu} t_{\nu \mu}^{*}\left(\mathbf{k}_{\|}\right) \psi_{\nu}^{-}\left(-\mathbf{k}_{\|}\right)
$$

Equations (B3) and (B4) impose a set of conditions on the values of scattering coefficients for the states with $-\mathbf{k}_{\|}$. Combined with the analogous conditions derived for the states with the incoming state in the right lead, they are compactly expressed as

$$
I=S\left(-\mathbf{k}_{\|}\right) S^{*}\left(\mathbf{k}_{\|}\right) \Rightarrow S\left(-\mathbf{k}_{\|}\right)=S^{T}\left(\mathbf{k}_{\|}\right) .
$$

The scattering matrix $S$ is defined as

$$
S=\left(\begin{array}{ll}
r & t^{\prime} \\
t & r^{\prime}
\end{array}\right)
$$

where $r^{(\prime)}$ and $t^{(\prime)}$ are matrices in the space of the lead modes and the primed coefficients describe scattering of the states incoming from the right. More specifically we have

$$
t_{\nu \mu}\left(-\mathbf{k}_{\|}\right)=t_{\mu \nu}^{\prime}\left(\mathbf{k}_{\|}\right) \text {and } r_{\mu^{\prime} \mu}\left(-\mathbf{k}_{\|}\right)=r_{\mu \mu^{\prime}}\left(\mathbf{k}_{\|}\right) \text {. }
$$

Equation (B7) gives

$$
T_{\mathcal{L} R}\left(-\mathbf{k}_{\|}\right)=\sum_{\nu \mu}\left|t_{\nu \mu}\left(-\mathbf{k}_{\|}\right)\right|^{2}=\sum_{\mu \nu}\left|t_{\mu \nu}^{\prime}\left(\mathbf{k}_{\|}\right)\right|^{2}=T_{\mathcal{R} L}\left(\mathbf{k}_{\|}\right) .
$$

In addition, for any two-terminal device, the Hermiticity of the scattering matrix guarantees that $T_{\mathcal{R} L}\left(\mathbf{k}_{\|}\right)=T_{\mathcal{L} R}\left(\mathbf{k}_{\|}\right)$(see Ref. 26) which finally proves the in-plane inversion symmetry mentioned at the beginning. The last step cannot, however, be taken for the partial (FS resolved) transmission probabilities. These quantities thus possess only the geometrical symmetry of the system.
*Present address: State Key Laboratory for Surface Physics, Institute of Physics, Chinese Academy of Sciences, P.O. Box 603, Beijing 100080, China.

'Present address: Max-Planck-Institut für Festkörperforschung, Heisenbergstrasse 1, D-70569 Stuttgart, Germany. Permanent address: Institute of Molecular Physics, P.A.N., Smoluchowskiego 17, 60-179 Poznań, Poland.

${ }^{1}$ K. M. Schep, P. J. Kelly, and G. E. W. Bauer, Phys. Rev. Lett. 74, 586 (1995).

${ }^{2}$ P. Zahn, I. Mertig, M. Richter, and H. Eschrig, Phys. Rev. Lett. 75, 2996 (1995).

${ }^{3}$ P. Weinberger, P. M. Levy, J. Banhart, L. Szunyogh, and B. Újfalussy, J. Phys.: Condens. Matter 8, 7677 (1996).

${ }^{4}$ K. M. Schep, J. B. A. N. van Hoof, P. J. Kelly, G. E. W. Bauer, and J. E. Inglesfield, Phys. Rev. B 56, 10805 (1997).

${ }^{5}$ P. Zahn, J. Binder, I. Mertig, R. Zeller, and P. H. Dederichs, Phys. Rev. Lett. 80, 4309 (1998)

${ }^{6}$ J. B. A. N. van Hoof, K. M. Schep, A. Brataas, G. E. W. Bauer, and P. J. Kelly, Phys. Rev. B 59, 138 (1999).

${ }^{7}$ J. M. MacLaren, X.-G. Zhang, W. H. Butler, and X. Wang, Phys. Rev. B 59, 5470 (1999).

${ }^{8}$ J. Kudrnovský, V. Drchal, C. Blaas, P. Weinberger, I. Turek, and P. Bruno, Phys. Rev. B 62, 15084 (2000).

${ }^{9}$ K. Xia, P. J. Kelly, G. E. W. Bauer, I. Turek, J. Kudrnovský, and
V. Drchal, Phys. Rev. B 63, 064407 (2001).

${ }^{10}$ I. Riedel, P. Zahn, and I. Mertig, Phys. Rev. B 63, 195403 (2001).

${ }^{11}$ J. Taylor, H. Guo, and J. Wang, Phys. Rev. B 63, 245407 (2001).

${ }^{12}$ M. Brandbyge, J. L. Mozos, P. Ordejón, J. Taylor, and K. Stokbro, Phys. Rev. B 65, 165401 (2002).

${ }^{13}$ V. Drchal, J. Kudrnovský, P. Bruno, P. H. Dederichs, I. Turek, and P. Weinberger, Phys. Rev. B 65, 214414 (2002).

${ }^{14}$ D. Wortmann, H. Ishida, and S. Blügel, Phys. Rev. B 65, 165103 (2002).

${ }^{15}$ D. Wortmann, H. Ishida, and S. Blügel, Phys. Rev. B 66, 075113 (2002).

${ }^{16}$ P. Weinberger, Phys. Rep. 377, 281 (2003).

${ }^{17}$ K. S. Thygesen, M. V. Bollinger, and K. W. Jacobsen, Phys. Rev. B 67, 115404 (2003).

${ }^{18}$ P. Mavropoulos, N. Papanikolaou, and P. H. Dederichs, Phys. Rev. B 69, 125104 (2004).

${ }^{19}$ J. Mathon, Phys. Rev. B 55, 960 (1997).

${ }^{20}$ J. Mathon, A. Umerski, and M. Villeret, Phys. Rev. B 55, 14378 (1997).

${ }^{21}$ E. Y. Tsymbal and D. G. Pettifor, J. Phys.: Condens. Matter 9, L411 (1997).

${ }^{22}$ S. Sanvito, C. J. Lambert, J. H. Jefferson, and A. M. Bratkovsky, Phys. Rev. B 59, 11936 (1999).

${ }^{23}$ J. Velev and Y. C. Chang, Phys. Rev. B 67, 144425 (2003). 
${ }^{24}$ J. Velev and W. H. Butler, Phys. Rev. B 69, 024404 (2004).

${ }^{25}$ Y. Imry, Introduction to Mesoscopic Physics, 2nd ed. (Oxford University Press, Oxford, 2002).

${ }^{26}$ S. Datta, Electronic Transport in Mesoscopic Systems (Cambridge University Press, Cambridge, England, 1995).

${ }^{27}$ D. S. Fisher and P. A. Lee, Phys. Rev. B 23, R6851 (1981).

${ }^{28}$ C. W. J. Beenakker, Rev. Mod. Phys. 69, 731 (1997).

${ }^{29}$ J. Kübler, Theory of Itinerant Electron Magnetism (Oxford University Press, Oxford, 2002).

${ }^{30}$ Because the magnetism of transition metals depends very sensitively on atomic structure (Ref. 29), it is important to know this structure quite accurately. The current drive to make devices whose lateral dimensions approach the nanoscale means that it is becoming increasingly important to know the atomic structures of these small systems microscopically while at the same time it is more difficult to do this characterization experimentally. It has become a practical alternative to determine minimum-energy structures theoretically by minimizing as a function of the atomic positions the total energy obtained by solving the Schrödinger equation self-consistently within the local density approximation (LDA) of density functional theory (DFT), thereby avoiding the use of any free parameters.

${ }^{31}$ P. Bruno, J. Magn. Magn. Mater. 121, 248 (1993).

${ }^{32}$ M. D. Stiles, J. Appl. Phys. 79, 5805 (1996).

${ }^{33}$ M. D. Stiles, Phys. Rev. B 54, 14679 (1996).

${ }^{34}$ M. D. Stiles and D. R. Penn, Phys. Rev. B 61, 3200 (2000).

${ }^{35}$ M. D. Stiles and D. R. Hamann, Phys. Rev. B 38, 2021 (1988).

${ }^{36}$ M. D. Stiles and D. R. Hamann, Phys. Rev. Lett. 66, 3179 (1991).

${ }^{37}$ K. M. Schep, P. J. Kelly, and G. E. W. Bauer, Phys. Rev. B 57, 8907 (1998).

${ }^{38}$ J. B. A. N.van Hoof, Ph.D. thesis, University of Nijmegen, Nijmegen, The Netherlands, 1997.

${ }^{39}$ J. B. A. N. van Hoof, K. M. Schep, P. J. Kelly, and G. E. W. Bauer, J. Magn. Magn. Mater. 177-181, 188 (1998).

${ }^{40}$ O. K. Andersen and O. Jepsen, Phys. Rev. Lett. 53, 2571 (1984).

${ }^{41}$ O. K. Andersen, O. Jepsen, and D. Glötzel, in Highlights of Condensed Matter Theory, edited by F. Bassani, F. Fumi and M. P. Tosi (North-Holland, Amsterdam, 1985), pp. 59-176.

${ }^{42}$ O. K. Andersen, Z. Pawlowska, and O. Jepsen, Phys. Rev. B 34, 5253 (1986).

${ }^{43}$ O. K. Andersen, O. Jepsen, and J. Šob, in Electronic Band Structure and its Applications, Vol. 283 of Springer Lecture Notes, edited by M. Yussouff (Springer, Berlin, 1987), pp. 1-57.

${ }^{44}$ I. Turek, V. Drchal, J. Kudrnovský, M. Šob, and P. Weinberger, Electronic Structure of Disordered Alloys, Surfaces and Interfaces (Kluwer, Boston-London-Dordrecht, 1997).

${ }^{45}$ T. Ando, Phys. Rev. B 44, 8017 (1991).

${ }^{46}$ K. Xia, P. J. Kelly, G. E. W. Bauer, A. Brataas, and I. Turek, Phys. Rev. B 65, 220401(R) (2002).

${ }^{47}$ M. Zwierzycki, Y. Tserkovnyak, P. J. Kelly, A. Brataas, and G. E. W. Bauer, Phys. Rev. B 71, 064420 (2005).

${ }^{48}$ K. Xia, P. J. Kelly, G. E. W. Bauer, and I. Turek, Phys. Rev. Lett. 89, 166603 (2002).

${ }^{49}$ M. Zwierzycki, K. Xia, P. J. Kelly, G. E. W. Bauer, and I. Turek, Phys. Rev. B 67, 092401 (2003).

${ }^{50}$ G. E. W. Bauer, A. Brataas, Y. Tserkovnyak, B. I. Halperin, M. Zwierzycki, and P. J. Kelly, Phys. Rev. Lett. 92, 126601 (2004).

${ }^{51}$ G. E. W. Bauer, Y. Tserkovnyak, A. Brataas, J. Ren, K. Xia, M. Zwierzycki, and P. J. Kelly, Phys. Rev. B 72, 155304 (2005).

${ }^{52}$ We follow the notation used in Ref. 42.
${ }^{53}$ O. K. Andersen and T. Saha-Dasgupta, Phys. Rev. B 62, R16219 (2000).

${ }^{54}$ R. W. Tank and C. Arcangeli, Phys. Status Solidi B 217, 89 (2000).

${ }^{55}$ O. K. Andersen, T. Saha-Dasgupta, R. W. Tank, C. Arcangeli, O. Jepsen, and G. Krier, Electronic Structure and Physical Properties of Solids: The Uses of the LMTO Method, Springer Lecture Notes in Physics, edited by H. Dreysse (Springer, New York, 2000), pp. 3-84.

${ }^{56}$ P. A. Khomyakov, G. Brocks, V. Karpan, M. Zwierzycki, and P. J. Kelly, Phys. Rev. B 72, 035450 (2005).

${ }^{57}$ J. Velev and W. H. Butler, J. Phys.: Condens. Matter 16, R637 (2004).

${ }^{58}$ C. Caroli, R. Combescot, P. Nozières, and D. Saint-James, J. Phys. C 4, 916 (1971).

${ }^{59}$ U. von Barth and L. Hedin, J. Phys. C 5, 1629 (1972).

${ }^{60}$ The larger and smaller lattice constants correspond to WignerSeitz atomic sphere radii of 2.669 and 2.621 Bohr atomic units, respectively.

${ }^{61}$ J. P. Perdew and A. Zunger, Phys. Rev. B 23, 5048 (1981).

${ }^{62}$ S. H. Vosko, L. Wilk, and M. Nusair, Can. J. Phys. 58, 1200 (1980).

${ }^{63}$ We adopt a "standard" configuration consisting of an fcc lattice with lattice constant of $a=3.614 \AA$, an $s p d$ basis, and von BarthHedin's exchange-correlation potential.

${ }^{64}$ P. Soven, Phys. Rev. 156, 809 (1967).

${ }^{65}$ P. Bruno, H. Itoh, J. Inoue, and S. Nonoyama, J. Magn. Magn. Mater. 198-199, 46 (1999).

${ }^{66}$ L. L. Henry, Q. Yang, W. C. Chiang, P. Holody, R. Loloee, W. P. Pratt, Jr., and J. Bass, Phys. Rev. B 54, 12336 (1996).

${ }^{67}$ H. A. M. de Gronckel, K. Kopinga, W. J. M. de Jonge, P. Panissod, J. P. Schillé, and F. J. A. den Broeder, Phys. Rev. B 44, 9100 (1991).

${ }^{68}$ C. Mény, P. Panissod, and R. Loloee, Phys. Rev. B 45, 12269 (1991).

${ }^{69}$ C. Kapusta, P. Fischer, and G. Schütz, J. Alloys Compd. 286, 37 (1999).

${ }^{70}$ Interface disorder can increase interface transmission if electronic structure mismatch leads to a highly reflecting clean interface. For example, for the majority-spin electrons at an $\mathrm{Fe} / \mathrm{Cr}$ (001) interface, this is the case. There, interface disorder in the form of two layers of $50 \%-50 \%$ alloy reduced the interface resistance by a factor of 3 (Ref. 9).

${ }^{71}$ S. Zhang and P. Levy, J. Appl. Phys. 69, 4786 (1991).

${ }^{72}$ S. F. Lee, W. Pratt, Jr., Q. Yang, P. Holody, R. Loloee, P. A. Schroeder, and J. Bass, J. Magn. Magn. Mater. 118, L1 (1993).

${ }^{73}$ T. Valet and A. Fert, Phys. Rev. B 48, 7099 (1993).

${ }^{74}$ W. P. Pratt, Jr., S. F. Lee, J. M. Slaughter, R. Loloee, P. A. Schroeder, and J. Bass, Phys. Rev. Lett. 66, 3060 (1991).

${ }^{75}$ M. A. M. Gijs, S. K. J. Lenczowski, and J. B. Giesbers, Phys. Rev. Lett. 70, 3343 (1993).

${ }^{76}$ J. Bass and W. P. Pratt, Jr., J. Magn. Magn. Mater. 200, 274 (1999).

${ }^{77}$ M. A. M. Gijs and G. E. W. Bauer, Adv. Phys. 46, 285 (1997).

${ }^{78}$ E. Y. Tsymbal, Phys. Rev. B 62, R3608 (2000).

${ }^{79}$ A. Shpiro and P. M. Levy, Phys. Rev. B 63, 014419 (2000).

${ }^{80} \mathrm{~S}$. Gerritsen, Master's thesis, University of Twente, Enschede, The Netherlands, 2002.

${ }^{81}$ P. A. Khomyakov and G. Brocks, Phys. Rev. B 70, 195402 (2004). 
${ }^{82}$ J. A. Støvneng and P. Lipavský, Phys. Rev. B 49, 16494 (1994).

${ }^{83}$ J. Cerdá, M. A. Van Hove, P. Sautet, and M. Salmeron, Phys. Rev. B 56, 15885 (1997).

${ }^{84}$ J. Mathon, Phys. Rev. B 56, 11810 (1997).

${ }^{85}$ W. H. Butler, X.-G. Zhang, T. C. Schulthess, and J. M. MacLaren, Phys. Rev. B 63, 054416 (2001).

${ }^{86}$ J. Mathon and A. Umerski, Phys. Rev. B 63, 220403(R) (2001).

${ }^{87}$ J. Mathon and A. Umerski, Phys. Rev. B 71, 220402(R) (2005).
${ }^{88}$ P. S. Krstić, X.-G. Zhang, and W. H. Butler, Phys. Rev. B 66, 205319 (2002).

${ }^{89}$ J. E. Inglesfield, J. Phys. C 14, 3795 (1981).

${ }^{90}$ S. Crampin, J. B. A. N. van Hoof, M. Nekovee, and J. E. Inglesfield, J. Phys.: Condens. Matter 4, 1475 (1992).

${ }^{91}$ G. D. Mahan, Many-Particle Physics, 2nd ed. (Plenum Press, New York, 1990). 